\title{
Dichtung als Propaganda? Die historisch-politische Ereignisdichtung zum Landshuter Erbfolgekrieg (1504/05)
}

\author{
Philipp Steinkamp
}

Als Herzog Georg der Reiche von Bayern-Landshut am 1. Dezember $15 \circ 3$ starb, entbrannte ein erbitterter Kampf um sein Erbe. Weil Georg über keinen männlichen Nachfolger verfügte, hatte er sein Herzogtum seiner Tochter Elisabeth und ihrem Gemahl Ruprecht, einem Sohn des Pfalzgrafen Philipp, vermacht. Diese Verfügung verstieß sowohl gegen das Reichsrecht, das eine weibliche Erbfolge ausschloss, als auch gegen den Hausvertrag der bayerischen Wittelsbacher, der bestimmte, dass beim Aussterben einer Linie im Mannesstamm deren Herzogtum unter den verbleibenden Linien aufgeteilt werden müsse. Aus diesem Grund erhob auch Albrecht IV. von Bayern-München, Oberhaupt der zweiten, im Jahre 1503 noch existierenden Linie, Ansprüche auf das Erbe Georgs des Reichen. Nachdem Vermittlungen König Maximilians erfolglos geblieben waren, kam es zum Krieg. Während sich Ruprecht und Elisabeth hauptsächlich auf die Hilfe des alten Pfalzgrafen und die Schlagkraft ihrer böhmischen Söldnertruppen verließen, hatte Albrecht eine eindrucksvolle Koalition geschmiedet. Auf seiner Seite zogen der Schwäbische Bund, Nürnberg und Brandenburg sowie die alten Feinde der Pfälzer, Württemberg und Hessen, in den Kampf. Albrechts wichtigster Verbündeter war aber Maximilian, dem der oberbayerische Herzog Landgewinne in Aussicht gestellt hatte und der die Gelegenheit nutzen wollte, um die Macht der Pfalzgrafen zu beschneiden. Der ein Jahr währende Krieg wurde von beiden Seiten mit äußerster Härte und unter Aufbietung sämtlicher Mittel geführt. Selbst als Ruprecht und Elisabeth starben, gaben ihre Parteigänger nicht auf und führten die Auseinandersetzung im Namen ihrer unmündigen Nachkommen weiter. Am Ende des Krieges standen furchtbare Verwüstungen in Bayern, der Oberpfalz und am Rhein sowie eine verheerende Niederlage der Pfälzer. ${ }^{1}$

1 Allgemein zum Landshuter Erbfolgekrieg: Würdinger (1868) Bd. 2, 174-279; Riezler (18781932 ) Bd. 3, 570-638; Ebneth und Schmid (2004); Bäumler (2005); Metz (2006). 


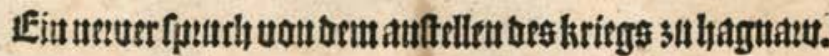

217idh freyt cint gesidst yes furmar thit an vr/adb gat oifan Jar Don bentunig bes beyligen reyd ITarinuliant bes furfter gleyd) Fitif in beves vino welfhen lanoen Dar fo groffe fach oút vnoer fanbet 21Tit gutem fryot vio cinigteyt In allen lanoent weyot veno pteyt Ir wiftes bet fid Gegefien

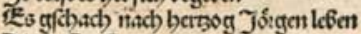
Diefurfan funbentrein freyt Das bat getvert nocb ber vilo feyt

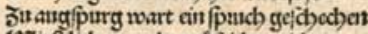

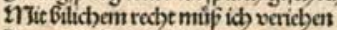
D.ts roart io fiefenlich geptocben Durd) gerechtigteyt if toas gerochat Danumb oas 2 bayerläot mat gef hat Jib $a 6$ gtenomen vinb ver6renot Ey tamat in peyn vno groffenot Berrog Z Zúpertte vergieng mit tobt Elifábetb ove furftin cyn

Mit in an junger furft follew

Dantadb oo fiengoer wifpect ant Ono wolt ban firften mider ftan Don mindyen berzog al6rabt ettent 2lud fain Bruber berbog wolf fü Er woltoas Gayerlanbetban gecibt Durch in furbt vil leyot varoabt Doch if es mirnit vaft layo Gotrech bie fach au 1 feyner fobays Das bie 23arm Den tumia banot veradht

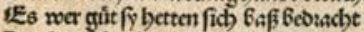
Das bat 8 bochwirbig furft gefochen an Sriberiats iftocs falben nam Żersog in 23 ayern vî P fatrograffipeym Der wolt io frum vit guttig forn (E) Cbet fich oer fach vnoer ftan Ono antgerieffic oic tunigtlich tron Das fich oer tunig wól er6amen 2Thaben fivot won weget oer amen 3erbog pbilips bat fich erge6at Im willen bes 2 Renrichen tunige lesen Twaferin giet fey ermeffon

Der jungen furfen foler nit vergeffen Der tunig foll Jr vater feyn Wenter fêl6 dic fesentein Etimant 3elieb oder3u laybet Das mentos balten bey trey ar vno aybe
23 rieff vnto figell bar v6er gefeat Docbetliden f tetten ift oas nit eben Dietrey6an fo groffen v6ernut 23 ey meyner treutes if nit guit 2fls baybect oic wil nit ban

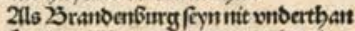
Berrog 2ll6ted)t bas int ver(ptochat Wirbes itr es wirot gerodit

Des gleyd) wentinty bat fid) gring

Dats fy feyen bar vont ofting

2Than barg in vent a a laffing erge6en Eelich in wat binteg if toas nit efas Gotbalt bie fach int eyner pfleg 217ich ounctt Die berren foyn bunveg Dy $06 \mathrm{an}$ ftet Die firzan nod 6 6en Wonin oer tunig eit wirbe gefiet Dam wentet fy willig vno geborfant feyt

Zaben bancl bie fiumen berren main keyn viit Teuburg futotnod in fpull

Oon wafferfurg ich (agen will Don 6raunaw veno lanbblit Ditgelfungen vno Gurshaufan gut

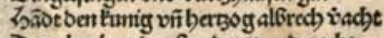
Des gleydan ben Guno tagt vno nadbe berrog fnibreyd oas ebd plit bat in verpotten bey lev6 vno guít Waben humig vno bersog $2016 \mathrm{r}$ adtet

Dito oen furften fptech in ir zkecht

Der rocr gef fraffic von feyner bantot Eein trey ftet in 3 up fantot Darumb bat Der tumig ganad)t ain firbe 27it fambet ber fiumen furferen gliot Das mant al frato yer wanodion Des bat oic tunigtlich) maicf tat 2lup gangen laffen irmanoat Jnallen / tetten pticf an gf chlagen Das einter ben anbern pl vertragent ITit wout vito werce grof v6amiut 23 if ber turigg oas ebcll plût 2luffipted bic fad nach gerechtigteyt Wan lanot vino leyot wirot 3 un getcyle Das wirot villeycht 6albt offanbar Got gee glict vito guttte Jar Das bo Eeley 6 ain gitter fitot Dnb allen Criftanlicben gitot Das Bit id) got 3 aller fart Jobbeyf Crift nezen6art

АBB. 4.1

Spruch von einem gewissen Crist Netzenbart (Gotha, Stiftung Schloss Friedenstein, Inv.-Nr. G. 35, 2/1925). 
Dass sich ein Konflikt von solchen Ausmaßen und solcher Intensität auch in der zeitgenössischen Publizistik niederschlug, kann nicht überraschen. So sind nicht weniger als 23 der Gattung der historisch-politischen Ereignisdichtung ${ }^{2}$ angehörende Texte überliefert, die den Landshuter Erbfolgekrieg thematisieren. ${ }^{3}$

In der Forschung wurden Werke dieser Gattung immer wieder als Propaganda bezeichnet, ohne dass der Begriff zuvor definiert und auf seine Verwendbarkeit hin überprüft worden wäre. ${ }^{4} \mathrm{Ob}$ aber ein Begriff, der seine heute gültige Ausprägung erst Anfang des letzten Jahrhunderts erhielt und meist auf Phänomene der Moderne bezogen wurde, tatsächlich eine spätmittelalterliche Literaturgattung adäquat bezeichnet, kann nicht von vornherein als ausgemacht gelten. ${ }^{5}$ Deshalb soll hier zunächst eine handhabbare Propagandadefinition vorgestellt werden, anschließend möchte ich am Beispiel der Dichtungen zum Landshuter Erbfolgekrieg zeigen, inwiefern die Bezeichnung „Propaganda“ das Phänomen Ereignisdichtung zu fassen vermag.

2 Grundlegend zur Gattung der historisch-politischen Ereignisdichtung: Völker (1981); Honemann (1997); Kerth (1997); Kellermann (2000). Zum Begriff „historisch-politische Ereignisdichtung" siehe dort S. 58-65.

3 Von Liliencron (1865-1869) Bd. 2, Nr. 232-248, S. 494-563; Bader (1885); Herrmann (1904); Lorentzen (1913) 209-218. Nicht zur Gattung der Ereignisdichtung ist ein nur sechs Verse umfassender Spruch zu zählen (Schäffer (1980)). Vgl. zu den kurzen Spottgedichten Honemann (1997) 402 und 418-419. Zu Überlieferung, älteren Editionen und Literatur vgl. Schanze (1985a). Dort finden sich auch Verweise zu den Artikeln namentlich bekannter Autoren. Beachte auch die entsprechenden Nachträge und Korrekturen in Bd. 11. Unediert blieben bislang: Ein kurzes Lied von Hans Schneider (Hamburg, SB u. U B, cod. hist. $31^{\mathrm{e}}, 390^{\mathrm{v}}$ ). Vgl. Schanze (1992) Sp. 791, Nr. II. 8; ein Spruch von einem gewissen Crist Netzenbart (Gotha, Schlossmuseum, Inv.-Nr. G. 35, 2/1925). Vgl. Schanze (2004); ein anonymer Spruch in einer Wiener Handschrift (Wien, Österr. Nat.bib., Cod. 2831 ${ }^{* *}$ ). Vgl. Bäumler (2005) Kat.-Nr. 3.38, S. 103104. Die Wiener Handschrift konnte leider nicht mehr berücksichtigt werden. Im Folgenden werden die edierten Texte mit dem abgekürzten Namen des Herausgebers (bei Liliencron mit der entsprechenden Nr.), unedierte mit dem Aufbewahrungsort der Überlieferungsträger bezeichnet.

Hingewiesen sei schließlich noch auf je eine bislang weitgehend unbekannte Fassung von Lor. und Lil. 233 in einer Münchener Handschrift (München, Bayerisches Hauptstaatsarchiv,

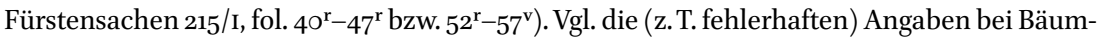
ler (2005) Kat.-Nr. 3.27, S. 95-97.

Das gesamte Textcorpus wurde bislang noch nicht umfassend untersucht. Eine kurze Zusammenschau der von Liliencron edierten Texte bietet Ehses (1880) 27-32. Eine Analyse eines Teils der Gedichte findet sich bei Kerth (1997) 117-121. Daneben ziehen sowohl Kerth wie auch Kellermann die Gedichte als Belegmaterial in den übergreifenden Kapiteln ihrer Untersuchungen heran. Vgl. Kerth (1997) Reg; Kellermann (2000) Reg.

4 Kerth bezeichnet die Ereignisgedichte als „Propaganda“, ((1997) 319-320); Kellermann als „Propagandadichtungen“, ((2000) 6); Völker als „agitatorische Propaganda“, ((1981) 18).

5 Zur Begriffsgeschichte: Schieder und Dipper (1984). 
Der Kommunikationswissenschaftler Gerhard Maletzke definiert Propaganda wie folgt: „,Propaganda' sollen geplante Versuche heißen, durch Kommunikation die Meinung, Attitüden, Verhaltensweisen von Zielgruppen unter politischer Zielsetzung zu beeinflussen. ${ }^{6} 6$ Um größere begriffliche Trennschärfe zu erreichen, möchte ich zudem den Begriff auf solche Beeinflussungsversuche beschränken, die darauf abzielen, ihr Publikum zu manipulieren - zum Beispiel durch den Einsatz gefälschter Fakten oder durch die Verwendung geeigneter rhetorischer Mittel. Beeinflussungsversuche, die mit sachlichen Argumenten operieren, ihre Informationsbasis zur Überprüfung offenlegen und auch Gegenargumente gelten lassen, also das Zielpublikum nicht zu manipulieren, sondern zu überzeugen suchen, sollen hingegen nicht als Propaganda bezeichnet werden.

Ob die Autoren mit ihren Texten eine Beeinflussung ihres Zielpublikums anstreben und ob sie zu diesem Zweck manipulative Mittel verwenden, soll zunächst an drei Gedichten, an einem Spruch über die Verhandlungen im Vorfeld des Krieges und an den beiden die Belagerung Kufsteins thematisierenden Liedern, untersucht werden. Anschließend wird das Textcorpus als Ganzes in den Blick genommen.

Ain spruch von herzogen Albrechten und vom pfalzgraven

Im Herbst des Jahres 1503 verschlechterte sich der Gesundheitszustand Herzog Georgs. Da seine Ärzte ihm zu einer Kur rieten, legte er die Regierung seines Landes in die Hände von sechs Kommissaren und machte sich auf den Weg ins Badische Wildbad. Doch viel weiter als bis nach Ingolstadt kam er nicht, dem Tod nahe kehrte er in die Stadt ein und rief Ruprecht, den Ehemann seiner Tochter Elisabeth, zu sich.

Bereits 1496 hatte der Herzog unter Absprache mit dem Pfalzgrafen Philipp in einem geheim gehaltenen Testament Elisabeth als Erbin seines Landes eingesetzt und verfügt, dass sie einen Sohn des Pfälzers ehelichen solle. 1499 wurde sie mit Ruprecht, dem dritten Nachkommen Philipps, vermählt. In den letzten Wochen seines Lebens versuchte Georg nun energisch, wenn auch letztlich erfolglos, den jungen Pfalzgrafen in sein Herzogtum einzusetzen und dessen Legitimation zu stärken. Zunächst verkündete der Fürst, er habe seinen Schwiegersohn, der bayerischen Geblüts sei, ${ }^{7}$ adoptiert. Anschlie-

6 Maletzke (1972) 157. Zum Stellenwert von Maletzkes Definition in der Propagandaforschung vgl. Bussemer (2005) 28.

7 Als Pfälzer gehörte Ruprecht zum Gesamthaus der Wittelsbacher. Darüber hinaus bestand 
ßend forderte er die Ingolstädter auf, dem Pfälzer zu huldigen, was ihm die Bürger aber verweigerten, da sie fürchteten, dass ihnen aus einer Parteinahme Schaden erwachsen könnte. Der Herzog ließ sich von diesem Rückschlag nicht beirren, ernannte Ruprecht zum Verwalter seines Landes mit allen Vollmachten eines Herzogs und übergab ihm die Städte und Schlösser Landshut und Burghausen, in die dieser am 23. und 27. November Einlass erhielt. Damit hatte Ruprecht die Hauptorte des Fürstentums und die dort deponierten Geldreserven des Landes unter seiner Kontrolle.

Georgs letzte Anordnung war die Einberufung eines Landtages nach Landshut, der auch stattfinden sollte, falls er zwischenzeitlich verstürbe. Tatsächlich ereilte ihn am 1. Dezember der Tod, den seine Räte wie zuvor verabredet einige Tage lang verheimlichten, damit die bereits begonnenen Rüstungsmaßnahmen für eine eventuelle kriegerische Auseinandersetzung mit Albrecht IV. weiter vorangetrieben werden konnten.

Der Herzog von Oberbayern hatte trotz aller Geheimhaltungsversuche schon früh von dem Testament Georgs erfahren und 1497 im Verborgenen bei König Maximilian eine Annullierung der Verfügung erwirkt. Nach dem Bekanntwerden von Georgs Tod wurde er unverzüglich bei dem Habsburger vorstellig und bat für sich und seinen formal an der Regierung beteiligten Bruder Wolfgang um die Belehnung mit Niederbayern. Diese gewährte ihm Maximilian nach längerem Zögern, allerdings nicht ohne Ruprecht ein Widerspruchsrecht einzuräumen.

Auf dem Landtag zu Landshut versuchten die beiden Kontrahenten, die Stände zu einer Anerkennung ihrer jeweiligen Ansprüche zu bewegen. Ruprecht, der persönlich anwesend war, ließ das Testament verlesen und verwies darauf, dass er mütterlicher- und väterlicherseits von bayerischer Abstammung sowie Adoptivsohn und Tochtermann Georgs des Reichen sei und dass dieser ihn zu Lebzeiten in sein Land eingesetzt habe. Albrecht und Wolfgang hingegen beriefen sich auf den Wittelsbachischen Hausvertrag. Unterstützt wurden sie in ihrem Ansinnen durch ihre Verbündeten und König Maximilian, der in einem Mandat die Stände bei Androhung der Acht dazu aufforderte, den Oberbayerischen Herzögen zu huldigen, da diese von ihm mit dem Fürstentum belehnt worden seien.

In der Landschaft herrschte große Uneinigkeit darüber, für welche Seite man sich entscheiden solle. Deshalb beschlossen die Stände zunächst, für die weiteren Verhandlungen mit den Parteien einen 64-köpfigen Ausschuss zu bilden.

über seine Mutter - eine Schwester Herzog Georgs - eine direkte Verbindung zur niederbayerischen Linie. 
Am 17. Dezember kam dieser überein, den König um Vermittlung zu bitten. Bis zu einem gütlichen Austrag sollte ein aus Vertretern aller Stände gebildetes Regiment die Herrschaft über das Fürstentum übernehmen. Ruprecht wurde das Versprechen abgenommen, derweilen auf jede Herrschaft zu verzichten, sich kriegerischer Handlungen zu enthalten und eine gütliche Einigung mit den oberbayerischen Herzögen zu suchen, oder, wenn dies nicht gelänge, einen Richtspruch der königlichen Majestät zu akzeptieren. Maximilian ergriff die Möglichkeit, die sich ihm durch die Unentschlossenheit der Landstände bot, und lud die Streitparteien für den 11. Januar nach Augsburg, um dort eine Einigung herbeizuführen oder ein Gerichtsurteil zu fällen. ${ }^{8}$

In den Kontext des Landshuter Landtages gehört das Gedicht Ain spruch von herzogen Albrechten und vom pfalzgraven (Lil. 232). Eingeleitet wird es, nicht ungewöhnlich für ein Ereignisgedicht, mit einer Klage über die allgemeine Schlechtigkeit der Welt, für die der anschließend geschilderte aktuelle Konflikt als Exempel fungiert: Die Verdorbenheit der Welt zeigt sich nach Auffassung des Dichters in der Verheimlichung des Todes Georgs, die „etlich tag“ (Lil. 232, 10) - tatsächlich waren es höchstens vier ${ }^{9}$ - angedauert habe und zum Nachteil der nächsten männlichen Erben, Albrecht und Wolfgang, gewesen sei. Des Weiteren manifestiere sie sich im Handeln einer anonymen Gruppe, welche sich über das Fürstentum ihres verstorbenen Herren "ain regiment angenomen“ (Lil. 232, 22) und geplant habe, einen "her von ferren“ (Lil. 232, 25), den Pfalzgrafen Ruprecht, dem kein Erbrecht zukomme, einzusetzen. Aus welchen Personen diese Gruppe bestand, erwähnt der Dichter nicht, doch wird aus den folgenden Versen und der Schilderung ihrer weiteren Taten deutlich, dass es sich bei ihnen um die Räte Georgs des Reichen handeln muss, die während der Abwesenheit ihres Herrn Statthalter in den beiden genannten Orten waren und nach dessen Tod Ruprecht in seinem Kampf um das Erbe unterstützten:

Si geprauchten sich übrigs gewalts

und gaben zů vil enthalts

zů Purkhausen und Landshůt

Lil. 232, 29-31

Der Darstellung des Dichters zufolge agierten die Räte selbstherrlich, anmaßend und aus eigenem Antrieb: Sie "gedeichten sich volkumen“ (Lil. 232, 21),

8 Vgl. zur Vorgeschichte des Erbfolgekrieges die oben genannte Literatur und Stauber (1993) $695^{-783}$.

9 Darauf verwies schon Liliencron (1865-1869) Bd. 2, Nr. 232, S. 495, Anm. zu V. 10. Vgl. auch Stauber (1993) $755^{-756 .}$ 
"geprauchten sich übrigs gewalts“ (Lil. 232, 29), trieben ihren „můtbillen [...]“ (Lil. 232, 39) und folgten „,irem aigen wellen“ (Lil. 232, 43). Ruprecht erscheint neben ihnen nur als ein Werkzeug, mittels dessen sie ihr selbstsüchtiges Ziel anstrebten, nämlich neben diesem „in irem gewalt zů pleiben“ (Lil. 232, 35) und sich am Land zu bereichern. Kerth schreibt zu dieser und zu ähnlichen Stellen in anderen Dichtungen zum Landshuter Erbfolgekrieg:

Über die erbrechtliche Ebene hinaus versuchen die Texte, Ruprecht den Niederbayern zu entfremden, indem sie ihn als ,Herren aus dem [sic] Fremde' darstellen, der nichts mit dem Herzogtum zu tun habe (Lil. 232-234). Die Ansprüche der pfalzgräflichen Familie, die sich immerhin auf Herzog Georgs (ungültiges) Testament und damit auf seinen erklärten Willen berief, sind als willkürlich oder niederen Motiven entstammend dargestellt. Die Schuld wird aber in den seltensten Fällen Herzog Ruprecht selbst angelastet: Fast immer sind es die Macht- und Besitzgier des alten Pfalzgrafen (zum Beispiel Lil. 234) und ein Stab böser, blutsaugerischer Ratgeber (zum Beispiel Lil. 232-234), denen die Schuld an der desolaten Lage gegeben wird. Hierbei wird wohl der [...] Tatsache Rechnung getragen, dass Ruprecht und seine Frau unter der Bevölkerung überaus beliebt waren. ${ }^{10}$

Letzteres wird aber kaum der Grund dafür gewesen sein, dass der Autor die Rolle der Räte so stark hervorhob, denn von einer besonderen Beliebtheit Ruprechts in der Bevölkerung kann mitnichten gesprochen werden, wie die wenig enthusiastische Reaktion der Landstände auf dessen Forderung, ihm zu huldigen, erkennen lässt.

Vielmehr dürfte der Dichter mit der Schuldzuweisung an die Räte den von Kerth erwähnten Zweck verfolgen, Ruprechts Ansprüche als unbegründet darzustellen, worauf die gesamte Schilderung des Konfliktes abzielt: Indem der Verfasser seine Darstellung erst mit den Ereignissen nach dem Tod Herzog Georgs und nicht schon mit dessen Erkrankung und seinen letzten Wochen in Ingolstadt beginnen lässt, kann er die Versuche des niederbayerischen Herzogs, seinen Schwiegersohn in sein Land einzusetzen und dessen Legitimation zu stärken - also dessen Adoption, die Aufforderung an die Ingolstädter, ihm zu huldigen, sowie seine Installation als Statthalter in Landshut und Burghausen -, übergehen. Die Tatsache, dass die Einsetzung Ruprechts dem letzten Willen Georgs entsprach und seine Räte ihm nur Gehorsam leisteten, wenn

$10 \quad$ Kerth (1997) 121. 
sie dem Pfälzer in Landshut und Burghausen Einlass gewährten und ihn in seinen Forderungen unterstützten, wird durch die Fehlinformation entstellt, einige namenlose Usurpatoren hätten Ruprecht installiert. Die Bezeichnung des Pfalzgrafen als „her von ferren“ (Lil. 232, 25) schürt einerseits Ängste vor dem landfremden Herrscher, der die lokalen Gewalten durch Vertraute aus seiner Heimat ersetzt und frei von persönlichen Bindungen das Herzogtum ausbeuten kann; und sie macht andererseits vergessen, dass das Geschlecht der Pfalzgrafen auch zum Gesamthaus der Wittelsbacher gehörte, Ruprecht über seine Mutter eng mit dem verstorbenen Georg verwandt war und er die Tochter des Landesherren geehelicht hatte. All dies diente Ruprecht aber als Legitimation und wurde auch von ihm und seinen Fürsprechern vor der versammelten Landschaft vorgebracht. Darüber hinaus wurde auch der Inhalt des im Spruch nirgendwo erwähnten Testamentes und damit der letzte Wille Georgs in Landshut öffentlich verlesen.

Da Ruprechts Ansprüche durch diese einseitige und stark verzerrende Darstellung des Konfliktes als völlig unbegründet erscheinen, wirken die im Folgenden erwähnten Gegenmaßnahmen Maximilians, Albrechts und Wolfgangs - das königliche Mandat und die Einlassungen der oberbayerischen Gesandten - umso gerechtfertigter.

Auch die in Landshut versammelte "frum landschaft" (Lil. 232, 58) habe, so der Dichter, gehandelt, um „zůvorkomen der selbigen list“(Lil. 232, 61). Sie hätte diejenigen, die diese Pläne verfolgten,

[...] vom Regiment getan, darzů erkiest ander person auß in von allen stenden, di alls dahin wenden, das den erben zů gehort;

Lil. 232, 63-67

weshalb sie zu loben seien. Wem nun das Land übertragen werden müsse, daran lässt der Dichter keinen Zweifel. Nachdem er im ersten Teil seines Gedichtes gegen eine Erbfolge durch Ruprecht agitiert hat, argumentiert er in der zweiten Hälfte für diejenige Albrechts und Wolfgangs: Es sei

[...] offenlich am tag,

daß niemant widersprechen mag,

daß die zwen fursten frumb

recht erben sind zů furstentumb,

als auch ir brief das klar sagen, 
di dann für sind getragen, darauf kunigliche majestat in gnädiglich gelichen hat.

Lil. 232, 77-84

Sodann fragt der Dichter sein Publikum: „Warumb wolt dann ain frum gemain/ in nit pillich huldung tůn?" (Lil. 232, 85f.). In den folgenden gut 80 Versen, die mit rhetorischen Fragen, Appellen und Warnungen gespickt sind, bemüht er sich, seine Rezipienten mit zusätzlichen Argumenten dazu zu bewegen, den oberbayerischen Brüdern zu huldigen. Zunächst appelliert er an ihr Ehrgefühl, wenn er ihnen vorhält, ihre Vorfahren seien der Obrigkeit stets gehorsam gewesen, weshalb „kain nation in geleich / sei gebest im ganzen reich“ (Lil. 232, 95f.). So sollten auch sie sich verhalten. Anschließend warnt er nachdrücklich vor den Konsequenzen, die eine Parteinahme für Ruprecht mit sich brächte. Diese würde zum Verderben des Landes führen, da die Herzöge von Oberbayern viele Verbündete hätten (vgl. Lil. 232, 76; 110; 118; 120; 165 f.). Damit bezieht sich der Verfasser geschickt auf eine der Hauptsorgen der Landstände, deren Taktieren im Erbfolgestreit vorrangig dem Ziel diente, diesen ohne Blutvergießen und Schäden für das Land beizulegen. Um eine gewaltsame Auseinandersetzung zu vermeiden, empfiehlt der Dichter, zunächst den Oberbayern zu huldigen, um dann den Streit gütlich beizulegen. Ruprecht solle nämlich durchaus das bekommen, was ihm gebühre: die „varende[.] hab“ (Lil. 232, 129).

Um Befürchtungen zu zerstreuen, dem Land könnten Nachteile aus dem Herrschaftswechsel erwachsen, schlägt der Dichter seinem Publikum vor, sie sollten, hätten sie „ainig geprechen, / di euch oder das ewer swechen“ (Lil. 232, 133f.), diese schriftlich festhalten und den beiden Fürsten vortragen; „alles das sich gepür / werd euch von in nit verzigen" (Lil. 232, 138f.).

Die letzten Argumente dürften vor allem das Ziel verfolgt haben, der handelund gewerbetreibenden Bevölkerung eine oberbayerische Erbfolge schmackhaft zu machen. Eine Landesunion brächte "gůten nutz und friden“ (Lil. 232, 151); man könnte, wie der Dichter anmerkt, Gewichte und Maße vereinheitlichen und ein gemeinsames Recht schaffen,

dann di gelerten sind zů swär, machen oft ainem den seckel lär;

si furren ainen ins decretal, piß ainer nicht mer hat überall.

Lil. 232, 159-162 
Nach einem letzten Hinweis auf die Gefahren, welche die Verweigerung der Erbpflicht beinhalte, schließt der Verfasser mit dem Wunsch: "got geb euch das pest in sinn!“ (Lil. 232, 168) seinen Spruch.

Die mit dem Werk verbundene Intention ist durch die zahlreichen Appelle leicht erkennbar: Die Rezipienten sollen dazu bewegt werden, Herzog Albrecht und seinem Bruder Wolfgang zu huldigen. Angesprochen werden aber nicht die Teilnehmer des Landtages, denen eigentlich die Entscheidung oblag - über sie wird ja in der dritten Person berichtet -, sondern die „landleit“ (Lil. 232, 97), womit vermutlich die nicht in Landshut anwesenden, niederbayerischen Landstände, vielleicht sogar alle Bewohner Niederbayerns gemeint waren. Ziel war es, in das durch den Tod Georgs entstandene Vakuum hineinzuwirken, um eine Entscheidung der unentschlossenen Landschaft zugunsten Albrechts und Wolfgangs herbeizuführen.

Zu diesem Zweck entwirft der Dichter eine klar gegliederte, stringente Argumentation, in der es ihm durch Unterschlagen von Tatsachen und Verzerrungen gelingt, alle die Gründe, welche der Pfalzgraf für sein Erbrecht anführte, verschwinden zu lassen. Darüber hinaus macht sich der Verfasser die Befürchtungen und Interessen seines Zielpublikums zunutze, um für seinen Standpunkt zu werben. Eine Parteinahme für die Pfälzer stellt er als schädlich für das Land dar: Die Räte wollten sich auf Kosten Niederbayerns bereichern, Ruprecht sei ein Herr aus der Fremde und ein Krieg unvermeidbar, nähme man ihn als Herrscher an. Als Argumente für eine Erbfolge durch Albrecht und Wolfgang wird vor allem auf den praktischen Nutzen einer Landesunion für die Untertanen verwiesen. Die rechtliche Begründung, der Hausvertrag und das Lehnsrecht, treten dabei in den Hintergrund.

Bei dem Autor handelte es sich vermutlich um einen Oberbayern, da er als Außenstehender zu seinem niederbayerischen Publikum spricht." ${ }^{11}$ Ob er aber im offiziellen Auftrag Albrechts IV. dichtete, ist fraglich, da dieser dem Vorschlag, Ruprecht die fahrenden Besitztümer zu überlassen - also auch Georgs sagenhaften Schatz und die enormen Getreidevorräte des Landes -, zu diesem Zeitpunkt wohl noch nicht zugestimmt hätte. Der Abschiedsgruß im vorletzten Vers, „Alde! ich var dahin“ (Lil. 232, 167), könnte darauf hinweisen, dass der Verfasser eine mündliche Verbreitung des Spruches durch einen reisenden Sprecher intendierte, doch ist bei der Deutung von solchen Formulierungen stets Vorsicht geboten. ${ }^{12}$ Sicherer lässt sich eine Publikation in der Form eines

11 Der Dichter/Erzähler vermeidet konsequent die 1. Person Plural.

12 Derartige Wendungen sind topisch, vgl. die von Liliencron beigebrachten Belege. Von Liliencron (1865-1869) Bd. 2, S. III. 
Einblattdruckes oder einer Flugschrift nachweisen, da das Gedicht in der handschriftlichen Überlieferung, mit einer für Kleindrucke typischen Datierung versehen ist: „1504 in novo anno“13.

Damit ist auch ein Zeitrahmen für die Abfassung des Textes gegeben. Das letzte erwähnte Ereignis ist die Wahl des Regimentes (vgl. Lil. 232, 64 f.), die am 29. Dezember erfolgte. Anfang des Jahres 1504 lag der Spruch ausweislich der oben zitierten Datierung in gedruckter Form vor und konnte in den offenen Konflikt hineinwirken. So schnell wie das Gedicht publiziert wurde, war es aber auch wieder überholt: Zwar bemühten sich die Parteien noch parallel zu den am 5. Februar in Augsburg beginnenden Verhandlungen um eine Huldigung der Landstände, doch ging dort die Entscheidungsbefugnis über das Erbe sukzessive von den Ständen auf den König über. Dieser versuchte, die beiden Fürsten auf eine Teilung des Erbes festzulegen und auch einige Besitzungen - sein sogenanntes Interesse - für sich herauszuschlagen. Letzteres hatte Albrecht IV. dem König bereits bei seiner vorläufigen Belehnung zugebilligt. Ruprecht aber war zu solchen Zugeständnissen nicht bereit und lehnte auch Maximilians Teilungsvorschläge ab. Als sich keine Lösung zu seinen Gunsten abzeichnete, verließ er die Verhandlungen, während seine Gemahlin den Krieg mit der Vertreibung des landständischen Regiments eröffnete. Der König stellte sich nun vollends auf die Seite der Oberbayern, sprach ihnen das gesamte Erbe zu und ächtete die Pfälzer.

Ein schönes lied von Kopfstain und das Benzenauer-Lied

Zu dem ,Interesse, das sich Maximilian gegenüber Albrecht IV. ausbedungen hatte, gehörte auch das Landgericht Kufstein. Ende Juni 1504 sandte der König einen seiner Räte dorthin, der von dem zuständigen Pfleger, Hans von Pienzenau, die Übergabe seines Schlosses erwirkte, wofür dieser in seinen Ämtern bestätigt wurde. ${ }^{14}$ Als aber am 9. August die Pfälzer die Stadt durch List einnahmen, öffnete der Pienzenauer auch ihnen die Festung - nach eigenen Angaben

\footnotetext{
13 Von Liliencron (1865-1869) Bd. 2, Nr. 232, S. 497. Auch Von Liliencron vermutete eine Druckabschrift.

14 Zur Eroberung Kufsteins und ihrer Vorgeschichte existiert eine reiche Spezialliteratur, hier seien nur die wichtigsten Arbeiten genannt: Höhlbaum (1887); Maretich (1888); Redlich (1888); Wiedemann (1895); Sinwel (1889); Schlagintweit (1903); Knöpfler (1904); Sinwel (1905); Lippott (1928) 28-33; Stelzer (1969); Wiesflecker (1971-1986) Bd. 3, 192-198; Rebitsch (2005); Haidacher (2005). Die Quellen sind bei Stelzer und Wiesflecker verzeichnet.
} 
am 15. August, anderen Angaben zufolge bereits nach einer Viertelstunde. Die Pfälzer beließen ihn auf seinem Posten, statteten ihn mit Proviant, Munition und Geschützen aus und legten eine aus böhmischen Söldnern bestehende Besatzung in die Stadt.

Erst nachdem Maximilian dem Gegner am Rhein und in Bayern schwere Niederlagen zugefügt hatte, konnte er eine Rückeroberung des Gerichts ins Auge fassen. Ende September erschien er mit gooo Mann vor Kufstein und forderte Stadt und Schloss zur Übergabe auf. Der Bürgermeister des Ortes, Permann, wollte dem Folge leisten, doch Hans von Pienzenau untersagte eine Kapitulation. ${ }^{15}$ Der darauffolgende Beschuss der Festung blieb ohne größere Wirkung, weshalb der König das Feuer zunächst auf die Stadt konzentrierte. Deren schwächere Verteidigungsanlagen waren den Geschützen nicht gewachsen und so musste der böhmische Stadtkommandant, nachdem er sich zum Verdruss Maximilians lange dagegen gesträubt hatte, am 12. Oktober Kufstein übergeben.

Der Pienzenauer aber glaubte in seiner Burg dem König trotzen zu können und verweigerte weiterhin die Kapitulation. Stattdessen forderte er vier Wochen Waffenstillstand - käme bis dahin kein Entsatz, wolle er die Festung öffnen. Auf eine von der Gegenseite angebotene Waffenruhe von zwei Wochen ging er nicht ein, und so einigte man sich nur auf eineinhalb Tage. Diese Zeit nutzte der Pfleger, um die Befestigungen des Schlosses zu verstärken, und Maximilian, um aus Innsbruck die beiden Riesengeschütze Purlepauß und Weckaufvon Österreich herbeizuschaffen. Als die Kanonen eintrafen, brach der Schlosskommandant den Waffenstillstand und nahm die Belagerer unter Beschuss. Er konnte aber nicht verhindern, dass die beiden Hauptstücke in Stellung gebracht wurden. Ihre Feuerkraft legte die Festung innerhalb von nur drei Tagen in Trümmer. Als der Pienzenauer nun um Gnade bat, war Maximilian nicht mehr bereit, sie zu gewähren, und befahl für den 17. Oktober die Erstürmung des Schlosses. In einem Akt der Verzweiflung wagte ein Teil der Burgbesatzung einen Ausfall, der Rest versuchte zu fliehen, doch alle wurden - entgegen dem Befehl Maximilians, keine Gefangenen zu machen von den königlichen Truppen in Ketten gelegt und abgeführt. Am nächsten Tag verhängte der König über die gesamte Besatzung das Todesurteil: Hans von Pienzenau und 18 seiner Männer wurden hingerichtet, etwa zwanzig weitere kamen nur deshalb mit dem Leben davon, weil Erich von Braunschweig und

15 Außer in der viel späteren Geschichtsschreibung wird die Auseinandersetzung zwischen Hans von Pienzenau und dem Bürgermeister nur in den beiden sich u. a. in diesem Punkt widersprechenden Liedern erwähnt (s.u.). 
einige weitere Fürsten aus dem Gefolge des Habsburgers im letzten Augenblick ihre Begnadigung erwirken konnten.

In der Literatur zur Eroberung Kufsteins nimmt die moralische und juristische Bewertung dieses Blutgerichts einen breiten Raum ein. Maximilians Vorgehen wird als "fürchterliche[n/s], ja barbarische[n/s] Strafgericht" und als „Akt übertriebener Härte" bezeichnet, ${ }^{16}$ während Rebitsch hervorhebt, dass sich der König mit seinem „fürchterlich[en]" Urteil durchaus im Rahmen des geltenden Kriegsrechts bewegte. ${ }^{17}$ Das Verhalten des Pienzenauers wertet die Forschung zwar fast einhellig als Verrat, doch bemühte sich namentlich die ältere bayerische und tirolische Geschichtsschreibung - letztere wohl unter Einfluss der damals dort sehr starken deutschnationalen Strömungen ${ }^{18}$ - den Schlosshauptmann zu entschuldigen: Als bayerischer Patriot habe er versucht, Kufstein vor dem Zugriff Österreichs und für die pfälzischen Wittelsbacher zu bewahren. ${ }^{19}$

Doch nicht erst in der modernen Forschung, sondern bereits unter den Zeitgenossen wurde um die Vorgänge in Kufstein heftig gestritten. Am 21. Oktober, also nur drei Tage nach der Hinrichtung des Pienzenauers und seiner Anhänger, ließen die pfalzgräflichen Hauptleute ein Schreiben an die königlichen Räte ausgehen, ${ }^{20}$ in dem sie zunächst ihre Parteinahme für den inzwischen verschiedenen Ruprecht und die Fortführung des Krieges nach seinem und seiner Gemahlin Tod rechtfertigen. Anschließend kommen sie auf die Hinrichtung des Pienzenauers und seiner Genossen zu sprechen: In bitterem Ton klagen sie, die Königliche Majestät habe ihre „freunde [...] mit etlichen knechten gefangen, dieselbigen unuerschuldt und allen redlichen ursachen enthaupten lassen. ${ }^{21}$ Sie hingegen hätten ihre Gefangenen stets „ritterlich und redlich gehalten. ${ }^{22}$ Sie mutmaßten, der König wolle sie mit seinem Strafgericht in Schrecken versetzen, damit sie von den Kindern Ruprechts und Elisabeths abrückten, was aber niemals geschehen solle. Den König bitten die Pfälzischen schließlich:

16 Wiesflecker (1971-1986) Bd. 3, 197. Ähnliche Formulierungen wählen auch Wiedemann: „barbarische Härte“ ((1895) 118), und Riezler: „antike Barbarei“ (1878-1932) Bd. 3, 624).

17 Vgl. Rebitsch (2005) 104-105. So auch schon Stelzer (1969) 15.

18 Vgl. Haidacher (2005) 95.

19 Am weitesten in der Apologie Pienzenauers geht Sinwel (vgl. Sinwel (1905) 16-27), obwohl er einige Jahre zuvor noch völlig anders über den Schlosshauptmann geurteilt hatte (vgl. Sinwel (1889) 8). Knöpfler argumentiert ähnlich, verzichtet aber darauf, ein abschließendes Urteil zu fällen, da dies die Quellenlage nicht zulasse (vgl. Knöpfler (1904) 14, 40) Vgl. auch Schlagintweit ((1903) 5).

20 Der Brief ist ediert bei Knöpfler (1904) 49-50.

21 Knöpfler (1904) 49-50.

22 Knöpfler (1904) 50. 
„bey sollicher handlung nit lenger beleyben, das ander teusch [sic!] nation von kaysern, königen, fursten, grauen, herrn, rittern und knecht nie mer geschehen noch gehört ist. “23

Im Namen Maximilians antworteten am 2. November seine Räte auf den Vorwurf, der König habe den Krieg anders als nach Herkommen der deutschen Nation geführt: „nun ist meniglich Wissen das in vil hundert Jaren nie erhört ist, das kain Teutscher gegen seinen Römischen Kong und Oberherrn gekriegt hab, dan ir. ${ }^{24}$ Um ein Exempel zu statuieren, habe er „weiter dan Kriegsrecht ist" gestraft. ${ }^{25}$ Zum Pienzenauer selbst und der Schlossbesatzung schreiben sie, die Königliche Majestät habe diese „ausser billichen Ursachen” verurteilt, da erstens der Pienzenauer "sein Glubt Brief und Sigil gegen Ir Majestet vergessen und sich verräterlich gehalten hat", womit die Übergabe der Festung an die Pfälzer gemeint ist, und da zweitens seine Gesellen nach der Übergabe der Stadt den Abzug versprochen hatten, dann aber doch zum Pfleger auf das Schloss geflüchtet waren. Zudem hätten sie Maximilian „etliche smeliche verachtliche Worte nachgeredt. “26

Die unterschiedlichen Positionen zum Kufsteiner Blutgericht spiegeln in gewisser Hinsicht auch die beiden Lieder Ein schönes lied von Kopfstain (Lil. 245) und das Benzenauer-Lied (Lil. 246 A, B, C). Da sie in ihrer Tendenz entgegengesetzt sind, aber einen ähnlichen Aufbau haben, sollen sie hier parallel zueinander analysiert werden.

Während das erste Lied mit einem Druck in zwei Auflagen aus der Offizin von Hans Schobser und zwei Handschriften gut, aber auch nicht außergewöhnlich reich überliefert ist, ${ }^{27}$ handelt es sich bei dem zweiten um das im 16. Jahrhundert am weitesten verbreitete „historische Lied ${ }^{428}$ : Mindestens sieben Drucke sind erhalten, die teilweise noch Jahrzehnte nach der Eroberung Kufsteins gefertigt wurden, als das Ereignis seine politische Bedeutung schon längst verloren hatte; es entstanden Übertragungen in die verschiedenen hochdeutschen Mundarten sowie eine niederdeutsche Fassung und sein Titel diente in der Folgezeit oft zur Tonangabe. ${ }^{29}$ Dies belegt eine weite zeitliche und räumliche Verbreitung sowie eine Popularität, die nur wenigen historisch-politischen Ereignisdichtungen beschieden war. Grundlage der folgenden Analyse ist Lil.

\footnotetext{
23 Knöpfler (1904) 50.

24 Der Brief ist in Zayners Chronik überliefert: Zayner (1763) 451-452.

25 Zayner (1763) 451.

26 Zayner (1763) 452.

27 Vgl. Schanze (1985a) Sp. 554.

28 Vgl. Herrmann-Schneider (1993) 156-157, Kat. Nr. 2.18; Ehses (1880) 31.

29 Vgl. Schanze (1985a) Sp. 555.
} 
A, da diese Fassung im Vergleich zu den anderen, die alle auf eine Redaktion zurückgehen, den wohl authentischsten Text bietet. ${ }^{30}$

Beide Dichtungen verzichten darauf, die Belagerung Kufsteins in den Kontext des Landshuter Erbfolgekrieges einzuordnen und die Vorgeschichte mit der Verpflichtung Pienzenauers dem König gegenüber und seinem Übertritt auf die Seite der Pfälzer darzustellen. In Lil. 245 beginnt die Schilderung der Ereignisse mit dem Zug des königlichen Heeres vor Kufstein und der Aufforderung Maximilians an die Stadt, sich ihm zu unterwerfen. Die Häufung der Worte "könig“ und „undertänig sein“ in der dritten Strophe erweckt den Eindruck, Maximilian fordere den Gehorsam ein, der ihm als Reichsoberhaupt zustehe, und macht vergessen, dass er weniger als König denn als Landesherr vor Kufstein zog, um seine territorialen Interessen durchzusetzen. Die Meinungsverschiedenheiten zwischen Hans von Pienzenau und dem Bürgermeister, die auf die Aufforderung des Königs, die Stadt zu übergeben, folgen, stellt der Verfasser in zwei antithetisch gebauten Strophen dar:

Der burgermeister was ein weiser man, er griff die sach nach dem besten an:

„die stat wöll wir aufgeben dem römischen könig wol in sein hand, so fristt er uns unser leben."

Der pfleger was ein stolzer man, er nam die sach nach dem bösten an, er wollt sich nit ergeben; hätt er dasselbig nit gethan, so hätt er behalten sein leben.

Lil. 245, 4-5

Der Bürgermeister ist „weise“, folgerichtig geht er „die sach nach dem besten an“, was hier bedeutet, die Stadt dem König zu übergeben, der dafür Gnade gewähren wird. Der Pienzenauer wird stattdessen als „stolz“ bezeichnet, er ist der Sünde der superbia anheimgefallen, und nimmt deshalb „die sach nach dem bösten an“, das heißt, er verweigert Maximilian seine Festung. Deshalb

30 Vgl. die Ausführungen von Hildebrand (1856) Nr. 9, 45-46 und die Anmerkungen; Von Liliencron (1865-1869) Bd. 2, Nr. 246 A, B, C, S. 556-557. Lil. 246 A hat eine inhaltlich notwendige Strophe (Str. 14), über die die anderen Fassungen nicht verfügen. Darüber hinaus bringt diese Fassung als einzige die Strophen 9-11 in der richtigen Reihenfolge. Vgl. Hildebrand (1856) Nr. 9, 49, Anm. zu Str. 10-11. 
habe er, so der Dichter, das Ende vorwegnehmend, sein Leben verloren. „Diese Wendung, einen Charakterzug vorauszuschicken, um eine folgende That oder Äußerung zu begründen, ist formelhaft, gehört zum epischen Apparat der Sänger “31 und entspricht sicherlich auch der mittelalterlichen Vorstellung vom Zusammenhang von Charakter und Handeln. Sie erfüllt hier aber vor allem eine propagandistische Funktion: Da die Handlungsweise des Pflegers durch seinen Hochmut, seinen „stolz“, hinreichend erklärt wird, kann darauf verzichtet werden, die Sachlage im einzelnen darzustellen.

Das Benzenauer-Lied schildert die Ereignisse völlig anders: Von einer Aufforderung Maximilians, ihm die Stadt zu übergeben, die man als einen Gnadenerweis verstehen muss, da die freiwillige Übergabe immer auch eine Garantie für Leib und Leben der Belagerten beinhaltete, ist nicht die Rede. Grund für die Beratung der Bürger über eine Aufgabe der Stadt ist hier vielmehr der Beschuss durch den Gegner, der schon in vollem Gange ist: „man tet die maur zerschießen" (Lil. 246 A, 2,5). Der Pienzenauer ist bei der Beratung anwesend und verbietet die Übergabe, da Maximilian bereits seinen Tod beschlossen habe:

„er schwůr bei allen hailigen,

ich můß mein leben verloren han.“ Lil. $246 \mathrm{~A}, 3,7$ f.

Auf die Befürchtungen des Bürgermeisters Permann hin, der Macht des Königs nichts entgegensetzen zu können, gibt sich der Pfleger zuversichtlich, denn Kufstein hätte schließlich eine starke Festung. Doch Pienzenauer hatte nicht mit einer List Maximilians gerechnet:

Ain frid ließ er anstellen

biß auf den dritten tag,

das tet der könig mit listen,

hew! warumb tet er das?

Die potschaft was im kommen

und was im vor wol bekannt:

zwo püchsen solt man pringen,

Lil. 246 A, 7,5-8,3

$31 \quad$ Hildebrand (1856) Nr. 8, 42, Anm. zu Str. 4. 
Als der Hauptmann bemerkt, dass ihn der König hintergeht und neue Geschütze heranbringen lässt, fühlt er sich nicht mehr an den Waffenstillstand gebunden und beginnt wieder mit dem Beschuss der Belagerer.

In Lil. 245 ist von einer „list“, also von einem betrügerischen, hinterhältigen Verhalten ${ }^{32}$ seitens Maximilians, nicht die Rede. Der Verfasser wählt stattdessen einen positiv konnotierten Begriff, er spricht von einem „sinn“ (Lil. 245, 6,1), einem klugen Gedanken, ${ }^{33}$ den Maximilian gefasst habe, als er „vil guts geschütz [...] für Kopfstain bracht" (Lil. 245, 6,2). Dass dies während eines Waffenstillstandes geschehen ist, wie Lil. 246 hervorhebt und auch andere Quellen belegen, wird hier unterschlagen, von dem "frid“ ist erst in der nächsten Strophe die Rede: Etwas unmotiviert wird erwähnt, man habe einen Waffenstillstand geschlossen, der durch den Pienzenauer aber gebrochen worden sei (Lil. 245, 7). Dem betrügerisch eine Waffenruhe ausnutzenden Maximilian und dem legitim handelnden Pienzenauer des Benzenauer-Liedes stehen also in Lil. 245 ein klug handelnder König und ein den Waffenstillstand nicht respektierender Festungskommandant gegenüber.

Der nun folgende Beschuss der Festung mit den beiden Riesengeschützen Purlepauß $\beta$ und Weckauf wird in beiden Gedichten recht ähnlich beschrieben, Unterschiede zeigen sich aber erneut in der Schilderung des Belagerungsendes: Lil. 245 verschweigt das Kapitulationsangebot der Schlossbesatzung und dessen Zurückweisung durch den König, berichtet dafür aber in zwei Strophen von dem Fluchtversuch der Belagerten. Lil. 246 hingegen widmet der Bitte um freien Abzug durch zwei vom Schlosspfleger entsandte Knaben und der abschlägigen Antwort Maximilians ein Strophenpaar. Von einem Fluchtversuch ist hier nicht die Rede, stattdessen heißt es lapidar:

man [das königliche Heer] saumt sich auch nit lange, man ruckt im rauch hinan;

auch xliii war man vahen

Lil. 246 A, 14,3-5

Die Hinrichtung des Schlosspflegers und seiner Männer stellt der Dichter von Lil. 245 knapp und kommentarlos dar - die Begründung für die Strafe war ja schon zu Beginn des Liedes durch den „stolz“ des Pflegers, seinen Widerstand gegen die königliche Majestät, gegeben. In einer weiteren, leider schwer verständlichen und vielleicht verderbten Strophe wird das Blutgericht aber noch in den größeren Rahmen des Landshuter Erbfolgekrieges gestellt:

32 Vgl. Grimm (1854-196o) Bd. 12, Sp. 1066-1067.

33 Vgl. Grimm (1854-196o) Bd. 16, Sp. 1125. 
Herzog Albrecht ist ein weiser man, er griff die sach zu dem besten an, er ist dem krieg vil zu frumme; der weisen sind also vil der thoren und der thummen.

Lil. 245,15

Liliencron paraphrasiert sie wie folgt: „Der König weiß besser, wie man Frieden machen soll, als Herzog Albrecht, der, obschon man ihn den weisen nennt, doch zu milde ist. So sind manche Weise Thoren. ${ }^{34}$ Unter ironischer Anspielung auf Albrechts Beinamen ,der Weise' und seine unkriegerische Wesensart spricht sich der Verfasser - wenn Liliencrons Interpretation der Stelle zutrifft $^{35}$ - gegen Albrechts zurückhaltendes Vorgehen und damit für die von Maximilian vor Kufstein bewiesene Härte aus.

Statt dieser Strophe verfügt die in einer Handschrift Oswald Gabelkovers enthaltene Fassung über eine andere, die ausführt, dass dem Herzog Erich von Braunschweig einige Gefangene übergeben wurden. Da Gabelkover seine Abschrift erst in der zweiten Hälfte des 16. Jahrhunderts ${ }^{36}$ anfertigte und die anderen Textzeugen die zuvor zitierte Strophe aufweisen, dürfte es sich bei der Erich-Strophe um eine spätere Umarbeitung handeln - möglicherweise in Anlehnung an das populäre Benzenauer-Lied, denn dieses schenkt der Hinrichtung und dem Einschreiten des Braunschweiger Herzogs große Aufmerksamkeit:

Auf den Richtplatz geführt, verwahrt sich Hans von Pienzenau in einer Verteidigungsrede gegen den Vorwurf, er sei „ain glüblos [,treuloser'] man“ (Lil. 246 A, 15,4): Er habe „redlich tan“, denn er habe dem Herzog Ruprecht geschworen, und wäre nicht zu loben, wenn er das ihm anvertraute Schloss ohne Not an den Feind übergeben hätte (Lil. 246 A, 15,2-16,3). Er beruft sich also auf die Treue-

34 Von Liliencron (1865-1869) Bd. 2, 551, Anm. zu Str. 15.

35 Liliencron hat bereits angedeutet, dass der genaue Sinn der Strophe nicht mehr zu erschließen ist. Vgl. von Liliencron (1865-1869) Bd. 2, 551, Anm. zu Str. 15. Zweifelhaft erscheint vor allem, dass der Verfasser in dieser Strophe die Wendung „er griff die sach zu dem besten an" (Lil. 245, 15,2) ironisch meinen soll, da er doch zuvor mit den gleichen Worten das Verhalten des Bürgermeisters als vorbildlich gekennzeichnet hat (vgl. Lil. 245, 4,2). Für Liliencrons Deutung spricht aber eine bisher in diesem Zusammenhang nicht beachtete Stelle bei Füetrer: dem „krieg er [= Albrecht] aber zu frum was und güettig und ain schbärer man und nit ain kriegs man“ (Spiller (1909) 239). Der Herzog wird hier wie in Lil. 245 als „zu frum“ für den Krieg bezeichnet. Die Kopplung mit dem Ausdruck „güettig“ belegt, dass mit frumme in Lil. 245,15 tatsächlich gemeint ist, Albrecht habe nicht hart genug durchgegriffen.

36 Stälin (1878). 
verpflichtung seinem Herrn Ruprecht gegenüber, wie es auch die pfalzgräflichen Hauptleute in ihrem Beschwerdebrief an Maximilian taten. Die vorangehende Verschreibung des Pienzenauers gegenüber dem König und der Bruch dieser Verschreibung, den die königlichen Räte in ihrem Antwortschreiben als Grund für die Hinrichtung des Pflegers angeben, werden hier geflissentlich verschwiegen.

Da Maximilian eine Bürgschaft von 30000 Gulden für den Pienzenauer ablehnt - „[k]ain bitt mocht da nit helfen“" (Lil. 246 A, 17,1) -, bleibt dem Schlosshauptmann nichts anderes übrig, als sich in sein Schicksal zu fügen:
[...] „seid ich dann můß sterben, der lieb got můß sein walten! von aller Bairen wegen můß ich heut ain tapfers halten."<smiles></smiles>
„hab urlaub, liebe welte!
got gesegen dich, laub und gras!
nun hilft mich heint kain gelte
und wirt mir mmer baß."

$$
\text { Lil. } 246 \text { A, } 17,5^{-18,8}
$$

Diese Abschiedsworte, denen das Pienzenauer-Lied wohl zu einem nicht geringen Teil seine Popularität im sechzehnten und dann wieder im neunzehnten Jahrhundert verdankt, ${ }^{37}$ dürften zum größten Teil eine Erfindung des Autors sein, haben aber auch einen historischen Kern: So berichtet der Kölner Gesandte Heinrich Slebusch, der sich zur Zeit der Belagerung im Gefolge Maximilians aufhielt, in einem Brief an den Ratskanzler seiner Heimatstadt, „Pienzenauer habe vor seinem Tode gesagt, er wolle sterben als ein frommer Bayer für alle Bayern. ${ }^{38}$ Ritterliches Selbstverständnis und Patriotismus dem niederbayerischen Herzogtum gegenüber - als dessen neuen Herrn er wohl Ruprecht ansah - drücken sich in diesen Worten aus und wurden vom Dichter effektvoll in die Abschiedsrede eingefügt.

Nach der Hinrichtung von 18 Mann schreitet Erich von Braunschweig ein und bittet den König für die restlichen Gefangenen um Gnade. Darauf lässt der Dichter Maximilian antworten:

37 Im Jahre 1904 errichtete man dem Schlosshauptmann an seiner mutmaßlichen Hinrichtungsstätte ein Denkmal, das als Inschrift eben jene Verse aus dem Benzenauer-Lied trägt. Vgl. die Abbildung bei Sinwel (1905) 3 .

$38 \quad$ Nur indirekt zitiert bei Höhlbaum (1887) 37, Anm. 11. 
„wir schwern auf unsern aid,

wer für ain wölt pitten

dem gäb man ain packenstraich.“

Lil. 246 A, 20,2-4

Für diesen Ausspruch während der Hinrichtung gibt es keinen Quellenbeleg, wohl aber dafür, dass Maximilian ihn in anderem Kontext geäußert hat: Als die Verhandlungen mit dem böhmischen Stadtkommandanten über eine Kapitulation Kufsteins zunächst scheiterten, drohte der König jedem, der noch von einer "taidung" spreche, mit einer Ohrfeige. Das Motiv wurde also wahrscheinlich erst später auf die Bitte Erichs von Braunschweig bezogen, ob durch den Dichter des Benzenauer-Liedes oder schon durch seine Quelle, ist nicht mehr zu klären. ${ }^{39}$ Es wird aber eindeutig dazu verwendet, um Maximilian als einen unbarmherzigen Herrscher darzustellen. „Zorniklich“ (Lil. 246 A, 20,5) gibt er dem Herzog einen Schlag, den jener lachend entgegennimmt: Härte und Großmut werden hier einander kontrastierend gegenübergestellt.

Die Analyse der beiden Gedichte dürfte gezeigt haben, dass sie in ihrer Tendenz einander diametral gegenüberstehen. Lil. 245 stellt Hans von Pienzenau als einen hochmütigen Mann dar, der seinem König die Öffnung der ihm anvertrauten Festung verweigert, einen Waffenstillstand bricht und sich feige durch Flucht zu retten versucht, weshalb er, wie man gar nicht mehr anders urteilen kann, die Strafe verdient hat, die ihm schließlich zuteil wird. Im BenzenauerLied erscheint der Schlosspfleger stattdessen als ein Mann, der im Widerstand nur seine Treueverpflichtungen gegenüber seinem Herrn Ruprecht und seinem Land erfüllt. Maximilian erweist sich hingegen durch seine Taten als ein hinterlistiger und unnachgiebiger Mann. Er vereinbart einen Waffenstillstand mit der Absicht, neue Geschütze herbeizuschaffen, nimmt die Kapitulation der Schlossbesatzung nicht an, lässt sich auch nicht durch die Verteidigungsrede des Pienzenauers oder eine Bürgschaft erweichen und begnadigt die letzten Männer des Pflegers erst, nachdem er den Bittsteller, Erich von Braunschweig, erniedrigt hat.

Die Tendenz der Gedichte resultiert weniger aus einer expliziten Deutung des Geschehens durch den Dichter - eine abschließende Bewertung der Vorgänge vor Kufstein oder gar eine Verurteilung der einen oder der anderen Seite findet sich in keinem der beiden Lieder - als vielmehr aus der unterschiedlich pointierten Darstellung derselben Ereignisse. Indem Details unterschlagen oder hervorgehoben werden, erscheint das Handeln des Königs beziehungs- 
weise des Schlosshauptmannes in den beiden Liedern in jeweils anderem Licht: Zum Beispiel wird in Lil. 245 nicht erwähnt, dass Maximilian den Waffenstillstand nutzt, um sich zu verstärken, was wiederum im Benzenauer-Lied hervorgehoben wird. So erzeugen die Autoren in den Köpfen der Rezipienten ein Bild von der Belagerung Kufsteins, das im Fall von Lil. 245 die königliche Partei, im Fall des Benzenauer-Liedes die pfälzisch-niederbayerische Partei in ihren Handlungen gerechtfertigt erscheinen lässt.

Die Angaben, welche die Verfasser in den letzten Strophen ihrer Lieder über sich machen, bestätigen die aus der Darstellung erschlossene Parteizugehörigkeit. Der Dichter von Lil. 245 bezeichnet sich als „ein freier lanzknecht gut“ (Lil. 245, 16,4), gehört also dem Heer Maximilians an. Möglicherweise ist er mit Hans Gern von Ems, dem Autor eines weiteren Gedichtes zum Landshuter Erbfolgekrieg (Lil. 241) identisch, da das Lied zur Belagerung Kufsteins, wie der Einblattdruck auch ausdrücklich vermerkt, „in behamer schlachtweise“, dem Ton von Lil. 241, abgefasst wurde. ${ }^{40}$ Die Wiederverwendung eines bereits im Laufe des Erbfolgekrieges benutzten Tones muss programmatisch verstanden werden. Er erzeugte beim Rezipienten die Erwartung, wie schon in Ain Lied von der behemschen schlacht, so auch hier den Bericht von einem glänzenden Sieg Maximilians zu erhalten. Auf diese Weise konnte die Wahrnehmung des Werkes bereits durch das Erklingen der Melodie gesteuert werden.

Der Autor des Benzenauer-Liedes behauptet, ein Adeliger zu sein, der der Belagerung auf dem Schloss beigewohnt habe; und "wär er nit entrunnen, / man het im auch drucken gschorn“ (Lil. 246 A, 22,7 f.). Seinen Namen möchte er aber nicht nennen - ein Umstand, auf den er ausdrücklich hinweist: „er tůt sich nit nennen / von wegen seiner stat" (Lil. 246 A, 22,3f.). Kellermann hat dieses explizite Verschweigen des eigenen Namens „ostentative[.] Anonymität" ${ }^{41}$ genannt. Auf diese Weise könnten die Autoren ihren Namen verbergen, um Verfolgungen wegen der von ihnen verfassten Gedichte zu entgehen, und gleichzeitig deren politische Brisanz herausstreichen. ${ }^{42}$ Hier ist der Fall allerdings etwas anders gelagert: Der Dichter verschweigt seine Identität nicht, weil er Sanktionen aufgrund des von ihm verfassten Liedes zu befürchten hätte, sondern seiner Zugehörigkeit zur Burgbesatzung wegen. Die „ostentative[.] Anonymität“ des Verfassers von Lil. 246 betont deshalb nicht die politische Brisanz seines Liedes, vielmehr hebt der Dichter damit die Brisanz des Ereignisses selbst hervor, indem er zum Ausdruck bringt, dass ihm Gefahr drohe, wenn er seinen

$40 \quad$ Vgl. Schanze (1985a) Sp. 554.

$41 \quad$ Kellermann (2000) 237.

42 Vgl. Kellermann (2000) 238. 
Namen nennt. Denn damit unterstellt er, Maximilian würde die Anhänger des Pienzenauers noch über das Ende der Belagerung hinaus verfolgen.

Ob der Autor aber wirklich ein Mitglied der Schlossbesatzung war, das im letzten Augenblick seinem Schicksal entrann, ist fraglich, da die feindliche Truppenpräsenz vor Kufstein eine Flucht unmöglich gemacht haben muss. Der Dichter berichtet denn auch seltsam distanziert über die Ereignisse. Intime Kenntnis der Vorgänge in der Festung, wie er sie als Augenzeuge eigentlich besessen haben müsste, offenbart er an keiner Stelle. Die Vermutung liegt deshalb nahe, dass der Autor hier eine Erzählerrolle einnimmt, mit der er die Brisanz des Ereignisses herausstreichen, sich als verlässlicher Zeuge der Darstellung präsentieren und seine Parteizugehörigkeit zum Ausdruck bringen kann.

Die beiden Ereignisgedichte spiegeln in ihren Tendenzen die Auseinandersetzung wider, die zwischen den Pfälzern und den Königlichen um die Frage entbrannte, ob die Hinrichtung des Pienzenauers gerechtfertigt war. Sie ergreifen für jeweils eine Seite Partei und versuchen diese durch die Schilderung ihrer Worte und Taten als rechtschaffend und ritterlich, die andere als verschlagen, hochmütig oder grausam zu erweisen. Dabei bleiben sie aber an der Oberfläche des Konfliktes, während etwa in dem Briefwechsel zwischen den pfälzischen Hauptleuten und den königlichen Räten mit den allgemeinen Kriegssitten beziehungsweise dem Eidbruch des Schlosspflegers argumentiert wird. Dennoch zeigen diese beiden Lieder, dass in der Gattung der historisch-politischen Ereignisdichtung nicht einfach nur zeitgeschichtliche Geschehnisse mit mehr oder weniger parteigetrübtem Blick wiedergegeben werden, sondern die Dichter mit ihren Texten auf aktuelle Streitfragen interessierten Bezug nahmen und auf den Verlauf der Diskussion einwirken wollten.

\section{Propaganda}

Nachdem an einigen Beispielen exemplarisch gezeigt werden konnte, dass die Verfasser von Ereignisgedichten versuchten, ihr Publikum zu beeinflussen, und zu diesem Zweck auch manipulative Mittel einsetzten, soll das gesamte Textcorpus überblicksartig betrachtet werden. Um die überlieferte Textmasse zu gliedern und um sich der Frage, welche Absichten die Dichter mit ihren Werken verfolgten, anzunähern, hat es sich als sinnvoll erwiesen, zunächst nach der von den Autoren verwendeten literarischen Form zu fragen.

Bei der Abfassung ihrerWerke konnten die Dichter zwischen zwei Grundformen wählen: zwischen dem aus Strophen aufgebauten und als Gesang vorgetragenen Lied und dem aus paarweise gereimten Versen bestehenden gespro- 
chenen Spruch. Die Forschung zur Ereignisdichtung vertritt bisher weitgehend die Ansicht, dass sich beide Formen hinsichtlich der verarbeiteten Stoffe, der Darstellungsweise und der Funktion nicht voneinander unterscheiden. ${ }^{43}$ Lediglich Gisela Ecker kommt bei ihrer Untersuchung der auf Einblattdrucken veröffentlichten Ereignisgedichte zu einem anderen Ergebnis. Sie schreibt:

Bei einem Vergleich der politischen Rede und dem historisch-politischen Lied können eine ganze Reihe von Unterschieden festgestellt werden. Sie liegen vor allem in der beschriebenen starken Festlegung der Lieder auf einige wenige Typen von Ereignissen und in ihrer Formelhaftigkeit begründet. Diese Einschränkungen bestehen für die Reden nicht; die politischen Reden sind thematisch weiter gefaßt [...], sie beziehen sich oft auf mehrere Ereignisse und häufig stärker auf die Zukunft und räumen in allen Fällen der Reflexion mehr Raum ein. ${ }^{44}$

Diese Einschätzung trifft auch auf die Lieder und Sprüche zum Landshuter Erbfolgekrieg zu: Für die Lieder gilt, dass sie sich alle auf ein einzelnes, abgeschlossenes und klar abgrenzbares Ereignis beziehen, auf eine Schlacht oder eine Belagerung; nur das fragmentarisch überlieferte Lied auf den ersten Feldzug der oberbayerischen Koalitionstruppen (Bad.) bildet insofern eine Ausnahme, da es eine Ereigniskette schildert. Die Rückbindung an den übergeordneten Konflikt, den Landshuter Erbfolgekrieg, ist häufig dürftig. In den meisten Fällen fehlt eine Erläuterung der politischen Hintergründe, die zu der besungenen Belagerung oder Schlacht geführt haben, oder sie bleibt völlig im Vagen (vgl. zum Beispiel Lil. 241). Fällt die Darstellung der Vorgeschichte doch einmal etwas ausführlicher aus, so bemüht sich der Dichter, den Erzählradius sukzessive einzuengen, um zu seinem eigentlichen Gegenstand vorzudringen (vgl. Lil. 238; Lil. 243).

Der Standpunkt des Verfassers lässt sich den Liedern größtenteils nicht direkt entnehmen. Erzählerkommentare betreffen überwiegend Details der beschriebenen Vorgänge, eine Gesamtdeutung des Geschehens am Ende der

43 Kellermann schreibt: Die „äußere Form der historisch-politischen Ereignisdichtung ist innerhalb der Versdichtungen unspezifisch; sie umfaßt mehrstrophige Lieder und Reimreden in Paar- oder Kreuzreim. [...] Damit ist eine Kategorisierung [...] nach strophischer und Reimpaardichtung, eben auch in Lied und Rede hinfällig." Kellermann (2000) 52 . Ähnlich urteilte auch Honemann: „Zwischen gesungenen und gesprochenen Texten zu differenzieren, erwies sich [...] nicht als sinnvoll, darüber hinaus auch nicht immer als möglich." Honemann (1997) 402.

Ecker (1981) Bd. 1, 228-129. 
Texte findet selten statt. ${ }^{45}$ Ihre Wirkung entfalten diese Dichtungen hingegen bereits durch die Darstellung des Ereignisses. Von zwei Ausnahmen abgesehen behandeln alle strophischen Dichtungen einen militärischen Vorfall, der für die eigene Partei siegreich ausgegangen ist. Die Verfasser hatten die Wahl, diese zu rühmen oder die gegnerische zu verspotten. Ein wichtiges Anliegen der Autoren war es daneben auch, die Unrechtmäßigkeit und Unehrenhaftigkeit des Gegners zu erweisen. Der beste Beleg dafür war der eigene Sieg - PaulGerhard Völker hat dies als das „einzig wirklich schlagende[.] Argument"46 bezeichnet. Die Autoren verfahren dabei stets auf dieselbe Weise. Zu Beginn der Darstellung lassen sie den Gegner in prahlerischen, vor Selbstbewusstsein strotzenden Reden die Überzeugung äußern, mit Leichtigkeit einen Sieg erringen zu können (vgl. zum Beispiel Her.; Lil. 247, 14f.). Der Ausgang des Geschehens, die Niederlage des Gegners, entlarvt dessen Rede anschließend als haltlos und den Redenden selbst als hochmütig, als jemanden, der seine eigenen Fähigkeiten überschätzt und sich so etwas anmaßt, was ihm nicht zusteht. Dies wiederum galt als eine Sünde, die unweigerlich eine Niederlage als Strafe nach sich ziehen musste. Hochmut und Niederlage stehen damit in einem wechselseitigen Folgeverhältnis: Einerseits ist die Niederlage Folge einer hochmütigen Einstellung; andererseits erweist erst die Niederlage die Einstellung als eine hochmütige.

Thematisierte der Dichter dagegen ein für die eigene Partei misslich ausgegangenes Ereignis, wurde dem Gegner Hinterlist vorgeworfen, wie es der Verfasser des Pienzenauer-Liedes tat. Weniger problematisch war die Sachlage bei der Plünderung des Klosters Waldsassen, die auch in einem Lied besungen wurde (Lil. 238). Da hier die Geschädigten wehrlose Mönche waren, brauchte man nicht dem Eindruck entgegenzutreten, die eigene Seite hätte eine Niederlage erlitten. Stattdessen konnte man leicht den Gegner verunglimpfen, indem man ihm Kirchenfrevel und Gräueltaten vorwarf.

Ebenso wie abschließende Deutungen fehlen in den meisten Fällen Appelle, die dem Publikum klare Handlungsanweisungen geben könnten. Gibt es sie doch, gehen sie nicht aus der Schilderung hervor, sondern werden bloß angehängt und vom Inhalt der Gedichte kaum unterstützt. So fordert zum Beispiel der Verfasser des Liedes über die Belagerung von Kaub eine gute Besoldung der an der Verteidigung der Stadt beteiligten Landsknechte (Her. 43-47). In der Schilderung der Kämpfe finden diese Landsknechte und ihre Leistungen aber

45 Dies hat bereits Honemann als charakteristisch für die Gattung der historisch-politischen Ereignisdichtung erachtet. Vgl. Honemann (1997) 407.

46 Völker (1981) 17. 
kaum Erwähnung, vielmehr konzentriert sich der Verfasser darauf, die militärischen Fehlschläge des Gegners herauszustellen und diesen so der Lächerlichkeit preiszugeben. Auch in dem Lied Lil. 236, das von der erfolglosen Belagerung Neumarkts durch die Nürnberger berichtet, unterstützt die Darstellung nicht die an den Pfalzgrafen gerichtete Bitte, die Stadt weiterhin zu beschützen. Überzeugender sind dagegen Lil. 241, in welchem nach der Schilderung des Sieges über die Böhmen davor gewarnt wird, den Krieg schon als gewonnen anzusehen, und Lil. 247, in dem andere Städte aufgerufen werden, es den Bürgern von Vilshofen gleichzutun, die sich einem pfälzischen Angriff tapfer erwehrten.

Das Fehlen einer abschließenden Deutung und klarer Handlungsanweisungen erschwert es, die mit der Abfassung der Lieder verbundene Intention zu ermitteln. Offenbar ging es weniger darum, die Hörer und Leser zu einer bestimmten Handlung aufzurufen, als die Deutungshoheit über ein Ereignis zu erlangen und auf diese Weise bestimmte Vorstellungen zu verbreiten. Stellte man den Gegner als unfähig, verworfen und schwach dar, die eigene Partei aber als erfolgreich, konnte das die eigenen Leute motivieren und Unentschlossene davon abhalten, sich dem Feind anzuschließen. ${ }^{47}$

Von den Liedern zum Landshuter Erbfolgekrieg unterscheiden sich die Sprüche deutlich. Ganz besonders trifft dies auf eine Gruppe von sechs Dichtungen zu. Sie stellen nicht wie die Lieder ein Ereignis dar, sondern erörtern konkrete Streitfragen und nehmen Stellung zum Erbfolgestreit als Ganzen. Neben dem oben analysierten Spruch Lil. 232 argumentiert noch ein weiterer im Vorfeld des Krieges für das Erbrecht Herzog Albrechts (Lil. 233). Ein Gedicht aus der Feder von Johann Kurtz ermahnt die Reichsöffentlichkeit, im Landshuter Erbfolgekrieg dem König zu Diensten zu sein (Lor.). Der anonyme Spruch Lil. 234 erschien nach Beginn der Kämpfe und klagt die Parteigänger Ruprechts einer Reihe von Schandtaten an, tadelt Städte, die sich dem Feind ergeben hatten, und ruft die unteren Volksschichten zum Widerstand auf. Der sehr umfangreiche Text Lil. 240 wendet sich gegen die niederbayerischen Räte und Hauptleute, erzählt, mit welchen Mitteln der junge Pfalzgraf angeblich an die Macht gekommen sei, und appelliert an Maximilian, endlich auf dem bayerischen Kriegsschauplatz aktiv zu werden. Das ungedruckte Flugblatt von Crist Netzenbart (Got.) schildert knapp Ursache und Verlauf des Krieges bis zu dessen weitgehender Einstellung und fordert einige renitente Städte auf, sich endlich zu fügen. Schließlich verkündet es das von König Maximilian im April 1505 erlassene Friedensgebot.

47 Honemann sieht die „ideologische Hauptfunktion der politischen Lieder und Sprüche [...] in der Sicherung und Festigung von bereits bestehenden Verständigungsgemeinschaften“ ((1997) 419). 
Diese Sprüche greifen - wie die oben untersuchten Reimpaardichtung - aus der Masse der Ereignisse diejenigen heraus, die ihnen für ihre Argumentation nützlich erscheinen. Sie formulieren klare Stellungnahmen zu dem Erbfolgestreit und wenden sich mit ihren Appellen an ein genau adressiertes Publikum. Sie wurden immer dann veröffentlicht, wenn der Konflikt an einen Wendepunkt geraten war und die Beeinflussung der Öffentlichkeit besonders dringlich erschien. Da präzise Handlungsanweisungen gegeben werden, ist es möglich, ihre Intention genau zu umreißen: Lil. 232 und Lil. 233 versuchten, die niederbayerische Bevölkerung zur Huldigung Albrechts und Wolfgangs zu bewegen, Lor., Lil. 234 und Lil. 240 beabsichtigen, die Öffentlichkeit zum Kampf gegen die Pfälzer zu mobilisieren, und das Gothaer Flugblatt bemüht sich darum, dem Friedensgebot des Königs Gehör zu verschaffen.

Neben den argumentierenden Texten existieren noch einige Reimpaardichtungen, die sich stärker der Schilderung von Ereignissen widmen. Von diesen erzählenden Sprüchen ähnelt am ehesten die Dichtung zur Belagerung Vilshofens (Lil. 248) den Liedern, alle anderen behandeln mehrere Vorkommnisse oder enthalten auch argumentierende Passagen: Hans Glasers Bericht über den Feldzug Ulrichs von Württemberg (Lil. 237) hat in seiner Aneinanderreihung von Ereignissen eher Ähnlichkeit mit einer Reimchronik als mit den strophischen Dichtungen. Hans Schneiders Spruch über die Feldzüge der Nürnberger (Lil. 235) thematisiert die einzelnen militärischen Aktionen seiner Heimatstadt, kommt daneben auch auf die Kriegstaten des Königs zu sprechen und verfügt über argumentierende Teile. Sein Spruch zur Böhmenschlacht (Lil. 244) konzentriert sich zwar nur auf ein Ereignis, stellt diesem aber eine ausführliche Einleitung voran, in der die Vorgeschichte des Konfliktes erläutert wird, und auch Johan Kurtz rückt seine Schilderung desselben Waffengangs in einen größeren politischen Zusammenhang (Lil. 242).

Die Autoren der Ereignisgedichte zum Landshuter Erbfolgekrieg wählten also je nach Inhalt und Intention unterschiedliche Formen. Recht fest umrissen war die strophische Dichtung, die nur ein einzelnes Ereignis aufnehmen konnte und sich hauptsächlich auf die Darstellung des Geschehens beschränkte, um eine Wirkung zu erzielen. Die Form des Spruchs besaß offenbar eine größere Flexibilität und konnte für Texte unterschiedlicher Art genutzt werden. Für Argumentationen eignete er sich besser als das Lied, dessen kurze, mit den Strophen übereinstimmende Sinneinheiten ${ }^{48}$ die Ausbreitung komplizierterer Gedankengänge nicht erlaubten. Ebenso dürfte in diesen Fällen

48 „Strophengrenzen [werden] nahezu einhellig zur inhaltlichen Zäsurierung genutzt." (Kellermann (2000) 303). 
der einfachere Versbau und der ruhigere Vortragston der Reimpaardichtungen den Bedürfnissen der Autoren eher entsprochen haben als die anspruchsvollere Strophenform und die Melodie der Lieder. Auch den Gedichten, die mehrere verschiedene Ereignisse oder Ereignisketten zum Inhalt hatten und zum Teil durch Zusätze laufend aktualisiert wurden (vgl. Lil. 235; Lil. 240), kamen Reimpaarverse eher entgegen als die Liedform mit ihrem auf Abgeschlossenheit hindrängenden Spannungsbogen.

Dass die Verfasser mit ihren Werken tatsächlich versuchten, die Meinungen, Attitüden und Verhaltensweisen des Publikums unter politischen Zielsetzungen zu manipulieren, dürfte deutlich geworden sein. Besonders die argumentierenden Sprüche versuchten das Publikum dazu zu bewegen, sich auf die Seite einer der Konfliktparteien zu stellen, und legten ihm bestimmte Handlungsweisen nahe. Die Lieder hingegen dienten wohl weniger dazu, direkte Handlungen zu provozieren. Sie sollten vermutlich eher die Wahrnehmung eines einzelnen Ereignisses steuern und Stimmungen erzeugen, um die Einstellungen der Rezipienten gegenüber den Parteien zu verändern.

Um diese Ziele zu erreichen, scheuten die Dichter auch nicht davor zurück, Fakten fortzulassen und zu verfälschen, wobei die Eingriffe unterschiedliche Ausmaße annehmen konnten. Die Texte Lil. 247 und Lil. 248 berichten weitgehend korrekt und ohne allzu große Übertreibungen über die Belagerung Vilshofens, wie der Vergleich mit einer unabhängigen Quelle beweist. ${ }^{49}$ Da die eigene Partei einen glorreichen Sieg davongetragen hatte, konnte hier auf grobe Verzerrungen verzichtet werden. Gab es aber Details, die die eigene Seite in ein schlechtes Licht rückten, wurden diese häufig einfach unterschlagen. Beispielsweise hebt jedes der fünf Gedicht zur Böhmenschlacht (Ham.; Lil. 241; Lil. 242; Lil. 243; Lil. 244) die - in der Tat - überragende Rolle Maximilians hervor, aber keines erwähnt, dass der König beim Ansturm auf den Gegner in Lebensgefahr geriet und vom Herzog von Braunschweig gerettet werden musste..$^{50}$ Noch weiter gingen die Verfasser der in Kapitel 2 und 3 untersuchten Werke. Hier nahmen die Dichter durch Auslassungen, Umstellungen und Umwertungen systematisch Manipulationen auf der Faktenebene vor, die eine Umdeutung des gesamten Geschehens zum Ziel hatten.

Neben Verfälschungen benutzten die Dichter auch zahlreiche rhetorische Mittel, um die persuasive Wirkung ihrer Texte zu erhöhen. Da hier kein vollständiger Katalog ausgebreitet werden kann, müssen einige Beispiele genügen: Von großer Bedeutung waren die Erzählerfigur, die das Geschehen kommen-

49 Vgl. den chronikalischen Bericht eines Stadtschreibers über dasselbe Ereignis:Wild (1979).

50 Vgl. hierzu Honemann (1997) 418. 
tierte, rhetorische Fragen und Appelle, mit denen das Publikum direkt angesprochen werden konnte, Ausrufe, Klagen und Anklagen, die dazu beitrugen, die Texte zu emotionalisieren, und nicht zuletzt ein bissiger, gegen den Feind gerichteter Spott.

Es lässt sich also festhalten, dass die Ereignisgedichte die Kriterien der Propagandadefinition insoweit erfüllen, als dass es sich bei ihnen tatsächlich um Versuche handelt, die Meinungen, Attitüden und Verhaltensweisen von Zielgruppen unter politischen Zielsetzungen zu manipulieren.

Eine wichtige Einschränkung muss aber gemacht werden. Die hier verwendete Definition weist den Zusatz auf, dass es sich bei Propaganda um "geplante“ Versuche handeln soll, Zielgruppen zu beeinflussen (beziehungsweise zu manipulieren), „[s]poradische, nicht geplante, aus einer Augenblickssituation sich ergebende ${ }^{“ 51}$ Versuche werden damit ausgeklammert. Eine Planung ist aber für die Ereignisgedichte zum Landshuter Erbfolgekrieg nicht auszumachen. Zwar hat die Forschung die These vertreten, dass hinter den Gedichten in vielen Fällen ein Auftraggeber gestanden habe, ${ }^{52}$ aber eine solche Initiative der Herrschenden, die zur Abfassung geführt hätte, lässt sich für die Dichtungen zum Landshuter Erbfolgekrieg nicht nachweisen, ja noch nicht einmal wahrscheinlich machen. Schon die Existenz zweier Dichtungen, die in Inhalt, Aufbau und Tendenz nahezu übereinstimmen (vgl. Lil. 247; Lil. 248), spricht gegen einen planenden Auftraggeber, denn dieser hätte seine Ressourcen sicherlich effektiver eingesetzt.

Die Produktion der Texte erfolgte wahrscheinlich weniger von oben nach unten in der Form eines klaren Auftrags, sondern umgekehrt von den Dichtern

$51 \quad$ Maletzke (1972) 156 .

$5^{2}$ Kellermann $((2000) 4,89)$ geht davon aus, dass die Dichter zum Teil aus Überzeugung, zum Teil im Auftrag ihre Werke verfassten. Honemann und Völker rechnen ebenfalls damit, dass ein Teil der Gedichte Auftragsarbeiten waren, letzterer gesteht den Verfassern aber dennoch zu, „mit ihrer eigenen Überzeugung hinter der Sache [zu stehen], weshalb sie auch kaum ihre politischen Einschätzungen und Parteinahmen wechseln." Vgl. Honemann (1997) 409, Anm. 5o (Hier auf die Sprüche zum Landshuter Erbfolgekrieg bezogen!), 415 und 417; Völker (1981) 26. Das Zitat 24. Kerth vermutet, dass Herrscher „nicht selten Bürger oder Berufsdichter beauftragt [haben], eine in Tendenz und Inhalt wohl genau vorgeschriebene Dichtung zu verfassen“, Parteigänger aber auch „von sich aus Propagandatexte im Sinne einer Kriegspartei“ gedichtet hätten (vgl. Kerth (1997) 272-279; die Zitate 273). In ihren jüngeren Untersuchungen vertritt Kerth die Ansicht, dass der größte Teil der Gedichte im Auftrag entstanden ist (vgl. z. B. Kerth (1999) 230). 
aus zu den Herrschern. Wilhelm Sunneberg sprach in seinem Werk Albrecht und dessen Gemahlin direkt an und erklärte, er habe in ihrem Dienst seine Kleider zerrissen (Lil. 233, 118-122). Offenbar dichtete und verbreitete er einen Spruch für seinen Fürsten, ohne von diesem beauftragt worden zu sein. Vielmehr trieb ihn die Hoffnung an, von dem Herzog eine Belohnung für seine propagandistische Tätigkeit zu erhalten. Da er aus Eigeninitiative agierte, war er wohl darauf angewiesen, den politischen Willen seines Herren zu antizipieren, und konnte nur hoffen, mit seinem Werk dessen Interessen zu entsprechen.

Neben einem fahrenden Berufsdichter wie Wilhelm Sunneberg, dem es in erster Linie um ein Geldgeschenk oder neue Kleider gegangen sein dürfte, ist auch ein Gelehrter wie Johann Kurtz anzutreffen, der wohl vor allem aus ideellen Gründen tätig war. Mit seinen Sprüchen zum Landshuter Erbfolgekrieg (Lor.; Lil. 242) wie auch mit seinen übrigen Ereignisgedichten ${ }^{53}$ versuchte er, die Politik König Maximilians publizistisch zu unterstützen. So forderte er die deutsche Nation auf, den Krieg im Inneren zu beenden und dem König im Kampf gegen seine europäischen Gegner beizustehen. Diese Gedanken zeigen eine große Nähe zu den politischen Ideen der Humanisten, von denen viele ohne besonderen Auftrag aber aus voller Überzeugung für das Reich und Maximilian eintraten. Auch Kurtz handelte vermutlich nicht in offiziellem Auftrag, denn als seinen Herrn nennt er nicht Maximilian, sondern Ulrich von Württemberg, der aber - obwohl er auf Seiten Albrechts und des Königs am Krieg teilnahm - in den Sprüchen nur am Rande auftaucht.

Engere Verbindungen zu einem Herrscher lassen sich im Falle des Spruchdichters Hans Schneider nachweisen. Zunächst war er gleichzeitig für Herzog Christoph von Bayern-München und Kaiser Friedrich III. tätig. Nachdem diese verstorben waren, trat er in den Dienst Maximilians. Selbstbewusst bezeichnete er sich als Sprecher der königlichen Majestät (vgl. zum Beispiel Lil. 244) und erscheint unter diesem Titel auch in Kölner Rechnungsbüchern. ${ }^{54}$ Nachdem er aber 1501 das Nürnberger Bürgerrecht erworben hatte, wird die Verbindung zu Maximilian nur noch lose gewesen sein, obwohl er auch in den Jahren danach nicht aufhörte, in dessen Interesse zu dichten. So verfasste er im Landshuter Erbfolgekrieg ein Lied und einen Spruch über den glänzenden Sieg des Habsburgers in der Böhmenschlacht (Ham.; Lil. 244). Dies bedeutet aber nicht, dass er ausschließlich für den König wirkte, denn Schneider berichtete in einem weiteren Werk auch über die Eroberungszüge seiner Heimatstadt Nürn-

53 Vgl. Schanze (1985b). Dort auch weitere Literatur.

54 Vgl. Pietzsch (1966) 81. 
berg. Ein Auftrag seitens der Nürnberger Obrigkeit hatte er dafür nicht, denn kurz nachdem das Gedicht erschien, wurden Schneider und seine Druckerin vom Rat dafür gerügt, dass sie einen Text ohne dessen Erlaubnis publiziert hatten. ${ }^{55}$

Die Bindung zwischen den Dichtern und der Obrigkeit wird also viel lockerer gewesen sein, als man bisher angenommen hat. Von einem geplanten Manipulationsversuch kann deshalb hinsichtlich der Ereignisgedichte keine Rede sein, die Initiative für die Werke lag im Wesentlichen bei den Dichtern selbst. Wollten die Fürsten gezielt auf die öffentliche Meinung Einfluss nehmen was im Landshuter Erbfolgekrieg häufig der Fall war - nutzten sie zumeist Medien, die ihnen vertrauter und leichter zugänglich waren, als die historischpolitische Ereignisdichtung. Dies waren vor allem die schriftlichen Erzeugnisse ihrer Kanzleien - Briefe, öffentliche Ausschreibungen, Mandate und Ähnliches - die mit Hilfe des eigenen Beamten- und Diplomatenapparates hergestellt und verbreitet werden konnten. ${ }^{56}$

Da die Obrigkeit als Auftraggeber nur eine geringe Rolle spielte, kommt dem Publikum als Bezugspunkt der Dichter eine größere Bedeutung zu. Viele Sprecher und Sänger heischten am Ende ihrer Gedichte nicht bei den Herrschenden um Lohn, sondern bei ihren Hörern (vgl. zum Beispiel Lil. 241, 17,4f.; Lil. 243, 15,8f.). Das Verfassen und Vortragen der Ereignisgedichte wurde demzufolge als eine Dienstleistung betrachtet, für die eine Vergütung erwartet werden konnte. Auch die Tatsache, dass ein großer Teil der Lieder und Sprüche zum Landshuter Erbfolgekrieg nicht nur mündlich, sondern zeitgleich auch als Flugblatt oder mehrblättrige Flugschrift verbreitet wurde ${ }^{57}$ belegt ein finanzielles Interesse der Produzenten. Denn Kleindrucke wurden in der frühen Neuzeit nicht kostenlos verteilt, sondern mussten käuflich erworben werden. ${ }^{58}$ Die zahlreichen Neuauflagen und Nachdrucke der Dichtungen zeigen, dass tatsächlich eine große Nachfrage nach den Texten bestand und die Rezipienten auch bereit waren, für diese zu zahlen.

Eine Planung und Steuerung der Propagandatätigkeit durch die Obrigkeit fand also wohl nicht statt, vielmehr handelten die Dichter der Sprüche und Lieder zum Landshuter Erbfolgekrieg aus Eigeninitiative. Dabei dürften kommerzielle Interessen neben den auch vorhandenen ideellen Beweggründen

55 Vgl. Schanze (1985a). Dort auch weitere Literatur.

$5^{6}$ Zahlreiche Beispiele für solche Texte finden sich bei von Krenner (1805).

57 Mindestens zwölf der 23 Ereignisdichtungen sind - z.T. mehrfach - im Druck erschienen, für einige weitere, nur handschriftlich überlieferte Texte ist dies zu vermuten. Vgl. die Angaben im Verfasserlexikon.

$5^{8}$ Auf den Warencharakter des Flugblattes hat besonders Schilling (1990) hingewiesen. 
von großer Bedeutung gewesen sein. Dies wirft aber weitere Fragen auf: Warum waren die Rezipienten bereit, für Propagandadichtung zu zahlen? Welche Bedürfnisse des Publikums konnten die Texte erfüllen, welchen Interessen kamen sie entgegen? Wurden die Dichtungen als Informationsquelle genutzt oder als Mittel zur Meinungsbildung? Konsumierten die Rezipienten die Publikationen der eigenen Partei, um sich die eigene Meinung noch einmal bestätigen zu lassen, oder suchten sie die Auseinandersetzung mit den Ansichten der Gegenseite? Und welche Rolle spielte der Unterhaltungsaspekt? Die Frage, welche Funktionen die Texte erfüllten, stellt sich damit erneut aus anderer Perspektive.

\section{Literatur}

Bader, T. 1885. Über ein auf der hiesigen Gymnasialbibliothek befindliches Fragment eines historischen Volkslieds aus dem Anfang des 16. Jahrhunderts. Programm Schleusingen 227: 3-24.

Bäumler, S. u. a. (Hg.) 2005. Von Kaisers Gnaden. 500 Jahre Pfalz-Neuburg. Katalog zur Bayerischen Landesausstellung 2005, Neuburg an der Donau, 3. Juni bis 16. Oktober 2005. Augsburg (Veröffentlichungen zur Bayerischen Geschichte und Kultur 50).

Bussemer, T. 2005. Propaganda. Konzepte und Theorien. Mit einem Vorwort von Peter Glotz. Diss. Erfurt. Wiesbaden.

Ebneth, R. und Schmid, P. (Hg.) 2004. Der Landshuter Erbfolgekrieg. An der Wende vom Mittelalter zur Neuzeit. Regensburg.

Ecker, G. 1981. Einblattdrucke von den Anfängen bis 1555. Untersuchungen zu einer Publikationsform literarischer Texte. 2 Bde. Göppingen (Göppinger Arbeiten zur Germanistik 314, zug. Diss. München 1978).

Ehses, S. 1880. Quellen und Literatur zur Geschichte des bayrisch-pfälzischen oder Landshuter Erbfolgekrieges 1504-1509. Diss. Würzburg.

Grimm, J. und W. 1854-196o. Deutsches Wörterbuch. 16 Bde. Leipzig.

Haidacher, C. 2005. Bilder und Texte zu 1504. In Von Wittelsbach zu Habsburg. Maximilian I. und der Übergang der Gerichte Kufstein, Rattenberg und Kitzbühel von Bayern an Tirol 1504-2004. Akten des Symposiums des Tiroler Landesarchivs Innsbruck, 15.16. Oktober 2004, hgg. C. Haidacher und R. Schober. Innsbruck (Veröffentlichungen des Tiroler Landesarchivs 12): 69-96.

Herrmann, F. 1904. Landsknechtslied auf die Belagerung von Caub 1504. Archiv für Hessische Geschichte und Altertumskunde N.F. 3: 113-122.

Herrmann-Schneider, H. 1993. Ain lyed vom bentznauwer. In Bayerisch-Tirolische G'schichten ... eine Nachbarschaft, Ausstellungskatalog Festung Kufstein 15. Mai bis 31. Oktober 1993, hgg. G. Ammann und M. Pizzinini, Bd. 1: Katalog. Innsbruck: 156-157, Kat.-Nr. 2.18. 
Hildebrand, H.R. (Hg.) 1856. Fr.L. von Soltau's Deutsche Historische Volkslieder. Zweites Hundert. Aus Soltau's und Leyser's Nachlaß und anderen Quellen. Leipzig.

Höhlbaum, K. 1887. Kölner Briefe über den bairisch-pfälzischen Krieg im Jahre 1504. Mittheilungen aus dem Stadtarchiv von Köln 4: 1-40.

Honemann, V. 1997. Politische Lieder und Sprüche im späten Mittelalter und der frühen Neuzeit. Die Musikforschung 50: 399-421.

Kellermann, K. 200o. Abschied vom, historischen Volkslied. Studien zu Funktion, Ästhetik und Publizität der Gattung historisch-politische Ereignisdichtung. Tübingen (Hermaea N.F. 9o, zugl. Habil. Berlin 1996).

Kerth, S. 1997. Der landsfrid ist zerbrochen. Das Bild des Krieges in den politischen Ereignisdichtungen des 13. bis 16. Jahrhunderts. Wiesbaden (Imagines medii aevi. Interdisziplinäre Beiträge zur Mittelalterforschung 1, zugl. Diss. Würzburg 1996).

Kerth, S. 1999. Der gute Grund. Modelle für Kriegsbegründungen in Liedern und Reimpaarsprüchen des 15. und 16. Jahrhunderts. In Der Krieg im Mittelalter und in der Frühen Neuzeit: Gründe, Begründungen, Bilder, Bräuche, Recht, hgg. H. Brunner. Wiesbaden (Imagines medii aevi. Interdisziplinäre Beiträge zur Mittelalterforschung 3): 229-262.

Knöpfler, J.F. 1904. Die Belagerung und Eroberung Kufsteins durch König Maximilian im Jahre 1504. Festschrift zur Erinnerung an die 4ooste Wiederkehr dieser denkwürdigen Tage im Auftrage des Stadtmagistrats Kufstein. Kufstein.

Von Krenner, F. (Hg.) 1805. Baierische Landtags-Handlungen in den Jahren 1429 bis 1513, Bd. 14: Nieder- und Oberländische Landtäge, im vereinigten Landshut-Ingolstädter Landantheile: Vom Tode Herzog Georgs 1503 anfangend biß zum allgemeinen Landesverein 1505 . München.

Von Liliencron, R. 1865-1869. Die historischen Volkslieder der Deutschen vom 13. bis 16. Jahrhundert. 5 Bde. Leipzig.

Lippott, E. 1928. Festung Kufstein. Ein Heimatbuch der Stadt und Festung. Kufstein (Tiroler Heimatschriften 3).

Lorentzen, T. 1913. Zwei Flugschriften aus der Zeit Maximilians I. Neue Heidelberger Jahrbücher 17: 139-218.

Maletzke, G. 1972. Propaganda. Eine begriffskritische Analyse. Publizistik 17: 153-164.

Maretich, G. Freiherr von Riv-Alpon 1888. Kaiser Maximilian I. vor Kufstein 1504. Organ der Militär-wissenschaftlichen Vereine 37: 159-196.

Metz, A. 2006. Das „Interesse“ des Königs. Maximilian I. und die niederbayerischen Landstände im Vorfeld des Landshuter Erbfolgekriegs. Zeitschrift für bayerische Landesgeschichte 69: 843-870.

Pietzsch, G. 1966. Fürsten und fürstliche Musiker im mittelalterlichen Köln. Quellen und Studien. Köln (Beiträge zur rheinischen Musikgeschichte 66).

Rebitsch, R. 2005. Die Eroberung der Festung Kufstein 1504. In Von Wittelsbach zu Habsburg. Maximilian I. und der Übergang der Gerichte Kufstein, Rattenberg und Kitzbü- 
hel von Bayern an Tirol 1504-2004. Akten des Symposiums des Tiroler Landesarchivs Innsbruck, 15.-16. Oktober 2004, hgg. C. Haidacher und R. Schober. Innsbruck (Veröffentlichungen des Tiroler Landesarchivs 12): 97-109.

Redlich, O. 1888. Zur Belagerung von Kufstein im Jahre 1504. мIÖG 9: 104-113.

Riezler, S. 1878-1932. Geschichte Baierns. 9 Bde. Gotha.

Schäffer, R. 1980. Ein bäuerlicher Spottvers auf König Maximilian I. aus dem Landshuter Erbfolgekrieg (1505). Zeitschrift für bayerische Landesgeschichte 43: 497-500.

Schanze, F. 1985a. Der Landshuter Erbfolgekrieg. In Die deutsche Literatur des Mittelalters. Verfasserlexikon, hgg. K. Ruh, Bd. 5. Berlin: Sp. 549-556.

Schanze, F. 1985b. Kurtz, Johann. In Die deutsche Literatur des Mittelalters. Verfasserlexikon, hgg. K. Ruh, Bd. 5. Berlin: Sp. 463-468.

Schanze, F. 1992. Schneider, Hans. In Die deutsche Literatur des Mittelalters. Verfasserlexikon, hgg. K. Ruh, Bd. 8. Berlin: Sp. 786-797.

Schanze, F. 2004. Netzenbart, Crist. In Die deutsche Literatur des Mittelalters. Verfasserlexikon, hgg. K. Ruh, Bd. 11. Berlin: Sp. 1047-1048.

Schieder, W. und Dipper, C. 1984. Propaganda. In: Geschichtliche Grundbegriffe. Historisches Lexikon zur politisch-sozialen Sprache in Deutschland, hgg. O. Brunner u. a., Bd. 5. Stuttgart: 69-112.

Schilling, M. 199o. Bildpublizistik der frühen Neuzeit. Aufgaben und Leistungen des illustrierten Flugblatts in Deutschland bis um 1700. Tübingen (Studien und Texte zur Sozialgeschichte der Literatur 29, zugl. Habil. München 1987-1988).

Schlagintweit, M. 1903. Kufsteins Kriegsjahre 1504, 1703, 1809. Eine Erinnerungs-Schrift zur 20ojährigen Wiederkehr der Erstürmung der tirolischen Grenzfeste durch die Bayern unter Kurfürst Max Emanuel. München (Darstellungen aus der Bayerischen Kriegs- und Heeresgeschichte 12).

Sinwel, R. 1889. Die Belagerung Kufsteins im Jahre 1504. Erweiterter Separat-Abdruck aus dem „Tiroler Grenzboten“. Im Anhange zweigleichzeitige Volkslieder und die Romanze von Anastasius Grün. Kufstein.

Sinwel, R. 1905. Hans v. Pienzenau der Schloßhauptmann von Kufstein im Jahre 1504. Erweiterter Separatabdruck aus dem „Tiroler Grenzboten“. Kufstein.

Spiller, R. (Hg.) 19og. Ulrich Füetrer, Bayerische Chronik. München (Quellen und Erörterungen zur Bayerischen und Deutschen Geschichte, NF, Bd. 2, Abt. 2).

Stälin, C.F. 1878. Gabelkover, Oswald G. In $A D B$, Bd. 8, 290-291.

Stauber, R. 1993. Herzog Georg von Bayern-Landshut und seine Reichspolitik. Möglichkeiten und Grenzen reichsfürstlicher Politik im wittelsbachisch-habsburgischen Spannungsfeld zwischen 1470 und 1505. Kallmünz/Opf. (Münchener historische Studien. Abteilung Bayerische Geschichte 15).

Stelzer, W. 1969. Die Belagerung von Kufstein 1504. Wien (Militärhistorische Schriftenreihe 12$)$.

Völker, P.-G. 1981. Das historische Volkslied. In Einführung in die deutsche Literatur des 
12. bis 16. Jahrhunderts, Bd. 3: Bürgertum und Fürstenstaat - 15./16. Jahrhundert, hgg. W. Frey u. a. Opladen (Grundkurs Literaturgeschichte): 12-37.

Wiedemann, T. 1895. Die Pienzenauer. Eine historisch-genealogische Abhandlung. Sonderabdruck aus dem IL. Bande des „Oberbayerischen Archives“ des Historischen Vereins von Oberbayern. München: 200-286; 347-407.

Wiesflecker, H. 1971-1986. Kaiser Maximilian I. Das Reich, Österreich und Europa an der Wende zur Neuzeit. 5 Bde. Wien.

Wild, K. 1979. Franz Seraph Scharrer und der Vilshofener Stadtschreiber Wolfgang Klopfinger. Studien zur Geschichte des Landshuter Erbfolgekrieges. Ostbairische Grenzmarken. Passauer Jahrbuch für Geschichte, Kunst und Volkskunde 21: 155-186.

Würdinger, J. 1868. Kriegsgeschichte von Bayern, Franken, Pfalz und Schwaben von 1347 bis 1506. 2 Bde. München.

Zayner, A. 1763. De Bello Bavarico Liber memorialis. In Rerum Boicarum Scriptores, hgg. A.F. Oefele, Bd. 2. Augsburg: 347-468. 